\title{
The Roles of Car Following and Lane Changing Drivers' Anticipations during Vehicle Inserting Process: A Structural Equation Model Approach
}

\author{
Li Li $\mathbb{D}^{1},{ }^{1}$ Dong Zhang $\mathbb{D}^{2},{ }^{2}$ Zhi-gang Xu ${ }^{\mathbb{D}},{ }^{3}$ Ping Wang, ${ }^{1}$ and Gui-ping Wang ${ }^{1}$ \\ ${ }^{1}$ School of Electronics \& Control Engineering, Chang'an University, Middle Section of Nan Er Huan, Xian, Shaanxi 710064, China \\ ${ }^{2}$ School of Transportation \& Logistics, Dalian University of Technology, No. 2 Linggong Rd., \\ Gan Jingzi District, Dalian, Liaoning 116024, China \\ ${ }^{3}$ School of Information Engineering, Chang'an University, Middle Section of Nan Er Huan, Xian, Shaanxi 710064, China
}

Correspondence should be addressed to Dong Zhang; zhangdong@dlut.edu.cn and Zhi-gang Xu; xuzhigang@chd.edu.cn

Received 17 May 2018; Revised 7 October 2018; Accepted 21 October 2018; Published 12 November 2018

Academic Editor: Stefano de Luca

Copyright (C) $2018 \mathrm{Li} \mathrm{Li}$ et al. This is an open access article distributed under the Creative Commons Attribution License, which permits unrestricted use, distribution, and reproduction in any medium, provided the original work is properly cited.

\begin{abstract}
Anticipating ability is a skill that drivers count on to handle risky tasks in the traffic. This paper explores how the drivers of lane changing vehicle and its immediately car follower anticipate surrounding vehicles' movements and adjust their manoeuvers during vehicle inserting process. The drivers' anticipating mechanisms are modelled in the framework of structural equation model and estimated from field data. Results show that the change of lane changing type or traffic signal affects the drivers' anticipation. Increased vehicle speed impels subject driver to anticipate driving condition in further future, but the stimulus is lower than the one coming from the kinematic comparisons of subject vehicle and other vehicles. The drivers care more about the vehicles' interactions with which they are personally involved than the one to which they are only onlookers. The drivers' responses to the counterpart vehicle's movements depend on the progress of vehicle insertion and their roles in vehicle interactions.
\end{abstract}

\section{Introduction}

A lane changing (LC) manoeuver is often accompanied by high collision risk, especially in congested traffic $[1,2]$. The mutual misunderstanding between the drivers of LC vehicle (LCV) and surrounding car following vehicles (CFV) is a major source of the risk. However, the cases of successful LC mostly happen in real traffic, when the drivers seem to be able to predict the risk and make adjustments in advance to avoid it. Such driving skill is usually named as anticipating ability or anticipation.

Driver's anticipation can reduce vehicle speed fluctuation and fuel consumption [3], but how this ability works and how to model it is still under study. Hofmann et al. [4] found that the anticipation gradually forms with driving experience, and some hints, such as turning light of the vehicle ahead, let the LCV driver know the counterpart's intention. Chan et al. [5] reported the weaker hazard anticipation of the novice drivers from the experienced drivers. Many existing LC decision models assume that the LCV's manoeuvre is impelled by a onetime and irrevocable decision of the vehicle driver, so the anticipation is often modelled as some predictive vehicle kinematic variables, such as vehicle gap or delay of travel time, measured at the moment of making LC decision $[6,7]$. However, the assumption has been challenged by the pieces of evidence obtained from recent empirical studies [8,9]. They found that the LCV's inserting process could last over ten seconds, and the preparation of such insertion is even longer. Some researchers have realized the risk of ignoring the continuity of LCV manoeuvre in traffic simulation, so they modelled the LCV's preparation movements before its insertion as a series of consecutive decisions followed by longitudinal manoeuvre adjustments [10-12]. In the models, the LCV driver's anticipation works continuously during the process of seeking an acceptable inserting gap in LC target lane. The LCV's longitudinal acceleration or deceleration was abstracted as the trade-off of driver's LC desire and his/her anticipated collision risk with surrounding vehicle if 
conducting LC $[13,14]$. It is noteworthy that most related studies are concerned with the LC preparing process on the original lane, but the anticipation should also work when the driver executes the inserting manoeuvre from the original lane to the target lane. Unfortunately, few efforts have been devoted to analyse anticipative inserting manoeuvre of the LCV driver [15].

More importantly, the anticipation is the behavioural basis of formulating LC model and CF model into a unified framework. Such integrated model can reduce the simulating error of many existing microscopic traffic flow models in the scenario when the LCV transits from the state of inserting preparation to the one of inserting execution [16, 17]. The modelling effort of CFV driver's anticipation in CF scenario has lasted for decades (see Section 2), but there rarely exist studies of how the skill works in LC scenario. In fact, the absence of CFV's anticipation could make potential conflict brought by LC manoeuver evolve to be a real collision.

To fill the existing research gaps, this study models and analyses the anticipating ability of the drivers of the LCV and its surrounding CFVs during the LCV's inserting process. The process is divided into two consecutive periods. The working mechanisms of the drivers' anticipation in two periods are formulated into two structural equation models (SEMs) correspondingly. A driver's anticipation to another vehicle is abstracted as a latent variable of the SEM. Vehicle trajectories and the indicators reflecting driving environment are utilized as the observable variables of the model. The transmission path of the drivers' anticipation on their behaviours and the influence degrees are identified based on the estimations of the SEMs.

The rest content is arranged as follows. Section 2 introduces the characteristics of driver's anticipation. Section 3 describes the modelling effort to figure out the working mechanism of the anticipation during the LCV's inserting process. Section 4 depicts data collection work. The model estimations are reported and discussed in Section 5. Section 6 concludes the main findings and points out the directions for future studies.

\section{Characteristics of Driver's Anticipation}

Figure 1(a) shows the minimum platoon involving a LC scenario, including a LCV, its following vehicle (FV), and leading vehicle (LV) on LC target lane. The LV's movement is assumed to affect the drivers of the LCV and the FV, but the LV driver does not suffer backward influences from the two following vehicles. The study period of this paper is the LCV's inserting process, and its detailed definition refers to the content in Appendix. The process can be divided into two stages by the entrance moment (EM, see Figure 1(a)), when body centre of the LCV crosses lane marking line. The period before the EM is anticipating period, while the one after the EM is relaxing period. The anticipations of the LCV driver and the FV drivers begin to work at the start of the anticipating period. Three driving relationships exist in each period. The FV and the LCV involve an LC relationship in anticipating period, and the CF relationships existing in this period include the following: (1) The FV follows the LV longitudinally on the target lane, and (2) the LCV follows the LV laterally on the original lane. In the relaxing period, the FV turns to follow the LCV laterally on target lane, and the other two CF relationships continue.

Anticipating ability of the drivers in the LCV and the FV assumes working in both longitudinal and lateral directions of lane traffic. This assumption has been verified by previous studies of CF behaviour. The longitudinal anticipation is modelled as a CF driver's estimation of one or more leaders' movements in one or more future moments [18-20]. Lateral anticipation is presented as that a staggered leader in the same lane or the neighbouring lane of the CFV brings the driver's lateral disturbance and triggers his or her deceleration [21, 22]. In some LC cases, the FV driver, who originally follows the LV in target lane, could anticipate adjacent LCV driver's inserting intention and decelerate voluntarily to create a larger inserting gap for the LCV [21]. Meanwhile, the LCV driver has a great possibility of anticipating the FV driver's decelerating intention and take advantage of the opportunity to finish the insertion. Under similar anticipation of the two drivers working simultaneously, they can coordinate their manoeuvers to ensure the vehicle safety during the LCV's inserting process. After the EM, the two drivers' anticipation continues working in the relaxing period. The LCV's relationship with the FV changes from the LC couple to a lateral CF couple, although its inserting manoeuvres continue after the EM. In this period, the two vehicles begin recovering their desired spacing with the LCV and the LV ahead, respectively [23-25]. Based on the previous findings mentioned above, the assumption of this study that the two drivers' anticipation works during the whole LCV's inserting process is reasonable.

Besides the nonexclusiveness and temporal continuity, other characteristics of the drivers' anticipation should be taken into account when modelling manoeuvres of the LCV and FV during the LCV's inserting process.

(1) Universality: driver's anticipations exist in every LC scenario. Sometimes it can be indirectly inferred from vehicle manoeuvers, while other times it only exists in the driver's mind. For instance, the FV driver's conservative anticipation to the adjacent LCV in a cooperative LC scenario can be inferred from observable predeceleration of the FV before the EM [26]. On the contrary, the FV driver who involves in a forced LC scenario has aggressive anticipation to the LCV. It induces the FV driver postponing the deceleration till or after the EM when the LCV forcibly but successfully is inserted into the target lane.

(2) Gradualness: each driver's anticipation is gradually formed during the LCV's inserting process as he or she continuously observes the other vehicles' movements. It is hard to imagine that a driver can accurately recognize the other driver's intentions by counting on the first impression only.

\section{Model}

3.1. Structural Equation Model. The characteristics of the driver's anticipation mentioned above belongs to the domain of human psychology. How to model and analyse the psychological influences on the manoeuvres of the LCV and the FV is the critical issue of this study. 


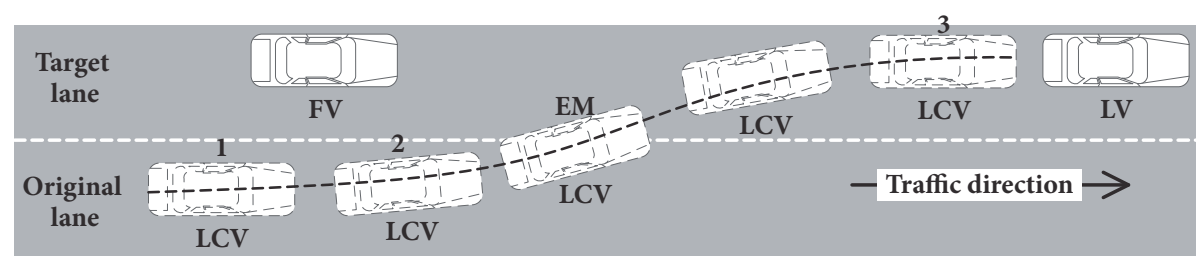

(a)

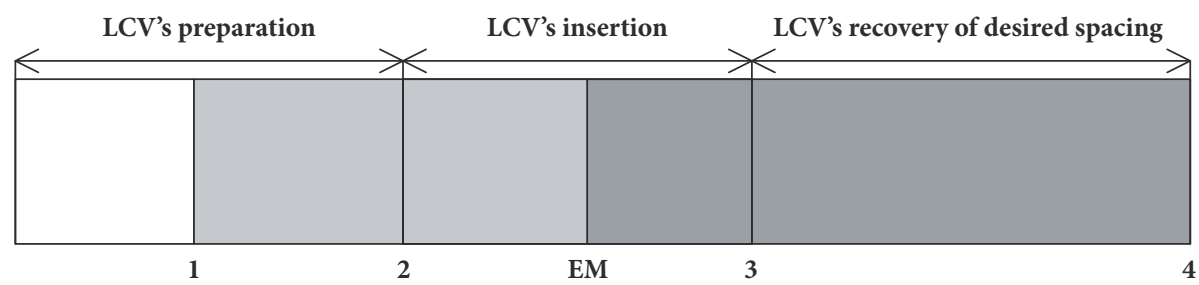

Anticipating period

Relaxing period

(b)

FIgURE 1: (a) A kind of LCV's insertion. (b) Timeline of the inserting process.

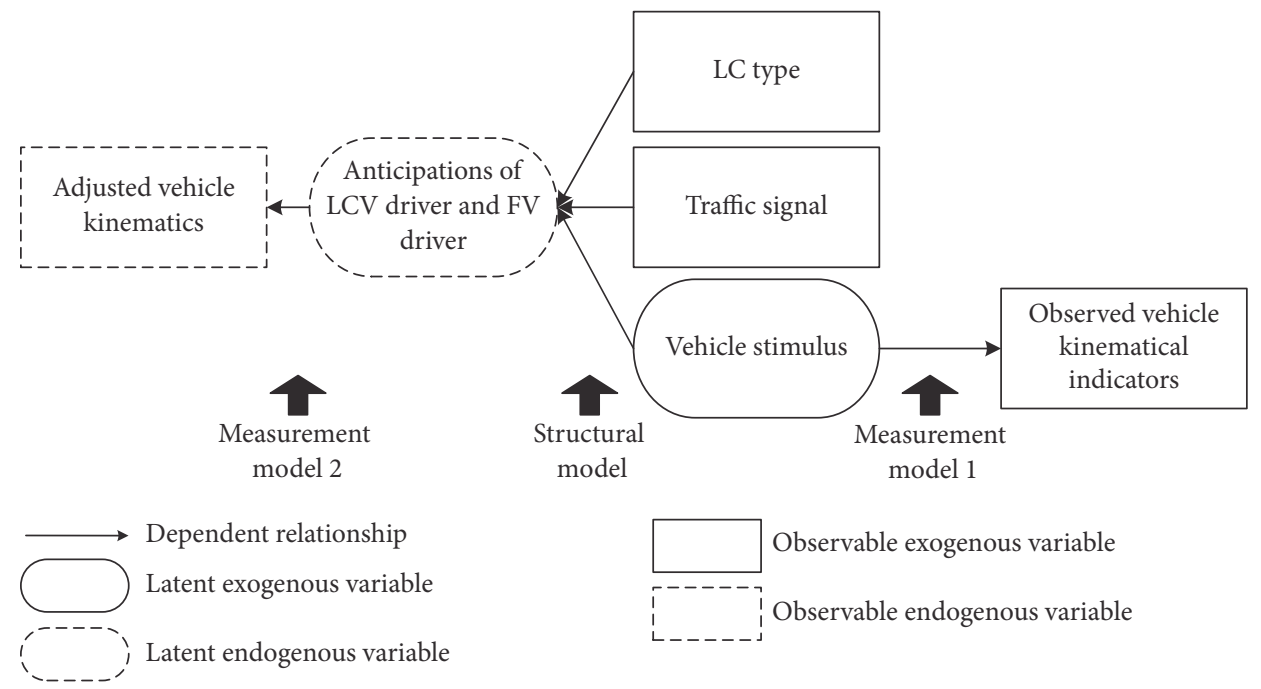

Figure 2: Conceptual model.

A conceptual model is proposed under the framework of SEM to depict how the psychological traits affect the drivers' behaviours. SEM provides an empirical approach to identify the cause-effect relationship that is formulated based on solid theories or empirical results [27]. It can identify the effects of personal risk perception and task loads on drivers' attitudes of driving safety [28]. The conceptual model consists of one structural model and two measurement models. The structural model hypothesizes the influential paths of the external stimuli to the drivers' anticipations, which could come from traffic signals, LC type, and other vehicles' movements. The vehicle stimuli can be inferred from some observed variables that reflect vehicle kinematics at the start of a study period.
This inferring system is formulated as the "measurement model 1" in Figure 2. The "measurement model 2" in the figure is another system to infer how the drivers adjust vehicle manoeuvers at the end of the period based on their anticipations.

The structural and measurement models are made of latent variables, observed variables, and their dependent relationships. They are illustrated as the normal rectangles, the rounded rectangles, and the directional lines in Figure 2. The dependent relationship presents as a directional line pointing from a causal variable to an affected variable. The anticipations of the LCV driver and the FV driver to future movements of surrounding vehicles are abstracted as the latent 
TABLE 1: Latent variable list.

\begin{tabular}{|c|c|c|c|}
\hline \multicolumn{2}{|c|}{ Endogenous latent variable } & \multicolumn{2}{|c|}{ Exogenous latent variable } \\
\hline Name & Meaning & Name & Meaning \\
\hline$L A_{F 2 P}$ & Anticipation of the FV driver to the LV & $L S_{L A F}$ & The LV stimulus for the FV driver \\
\hline$L A_{F 2 L C}$ & Anticipation of the FV driver to the LCV & $L S_{L C 4 F}$ & The LCV stimulus for the FV driver \\
\hline$L A_{L C 2 F}$ & Anticipation of the LCV driver to the FV & $L S_{L \& L C 4 F}$ & The LV\&LCV stimulus for the FV driver \\
\hline \multirow[t]{3}{*}{$L A_{L C 2 L}$} & Anticipation of the LCV driver to the LV & $L S_{L 4 L C}$ & The LV stimulus for the LCV driver \\
\hline & & $L S_{F 4 L C}$ & The FV stimulus for the LCV driver \\
\hline & & $L S_{L \& F 4 L C}$ & The LV\&FV stimulus for the LCV driver \\
\hline
\end{tabular}

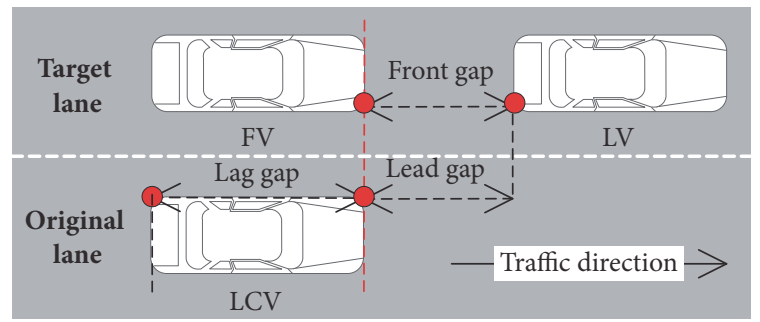

(a)

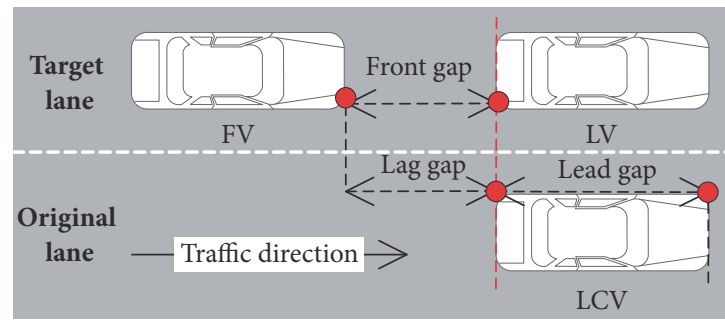

(b)

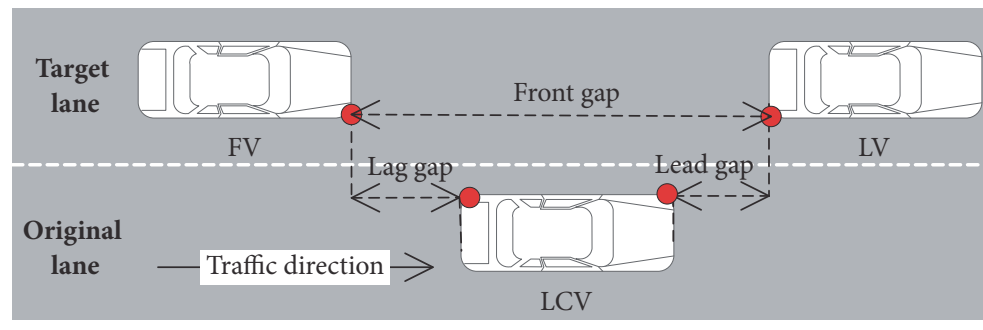

(c)

Figure 3: (a) Forward insertion. (b) Backward insertion. (c) Median insertion.

variables listed in Table 1, as they cannot always be observed from vehicle trajectories. The observed variables listed in Table 2 are defined from the view points of the LCV driver and the FV driver in specific scenarios. Their definitions will be detailed in Section 3.2.

\subsection{Variables}

3.2.1. Scenario-Dependent Variable. The LCV could be inserted into target lane in different ways [29], so three inserting scenarios illustrated in Figure 3 are defined in this study. In a forward inserting scenario, the LCV overtakes the FV to find opportunity of conducting the insertion. In a backward inserting scenario, the LCV lets the LV pass it and cuts in the backward gap. In a median inserting scenario, the LCV has run aside the gap between the LV and the FV for a while and finds a proper time to fill in the gap. The observed variables are measured at the start and the end of the two study periods. Their settings are defined in Table 2.

The settings of some variables at the start of the anticipating period depend on the observing point of each driver in the scenarios defined above. Here take the lag gap observed by the FV driver $\left(G_{\text {lag4F }}\right)$, for instance. In the forward inserting scenario (see Figure 3(a)), lag gap appears in the negative direction of the FV driver while in the positive direction of the LCV driver, it sets $G_{\text {lag } 4 F}^{1}<0$ and $G_{\text {lag } 4 L C}^{1}>0$. Note that the LCV driver needs to estimate the FV driver's reaction to the LV's movements, so the two drivers are assumed to stay in the same line when observing the front gap $\left(G_{\text {front }}^{1}\right.$ in Table 2$)$ at the start of the anticipating period. Similarly, the FV driver is assumed to observe the lead gap ( $G_{\text {lead }}^{1}$ in the table) as the LCV driver does.

3.2.2. Scenario-Independent Variable. Besides the scenariodependent variable, the values of some variables do not relate to the scenarios defined above. Front gap always appears in the positive direction of the FV in each scenario (see Figure 2), so it holds the positive value as an input or output of drivers' anticipations $\left(G_{\text {front }}^{1} / G_{\text {front }}^{\mathrm{EM}} / G_{\text {front }}^{n}>0\right.$ in Table 2). Speed difference is often used to measure the degree of driving safety. A driver can evaluate the collision risk to another vehicle from varying trends of their speed differences. For example, if the FV is slower than the $\operatorname{LV}\left(S D_{F \& L}^{1} / S D_{F \& L}^{E M} /\right.$ $S D_{F \& L}^{n}>0$ in the table), its head-end collision risk to the LV is lower, and vice versa. Hence, a positive speed difference between the two vehicles $\left(S D_{F \& L} / S D_{F \& L C} / S D_{L C \& L}\right.$ in the table) indicates a safer driving condition than the condition 


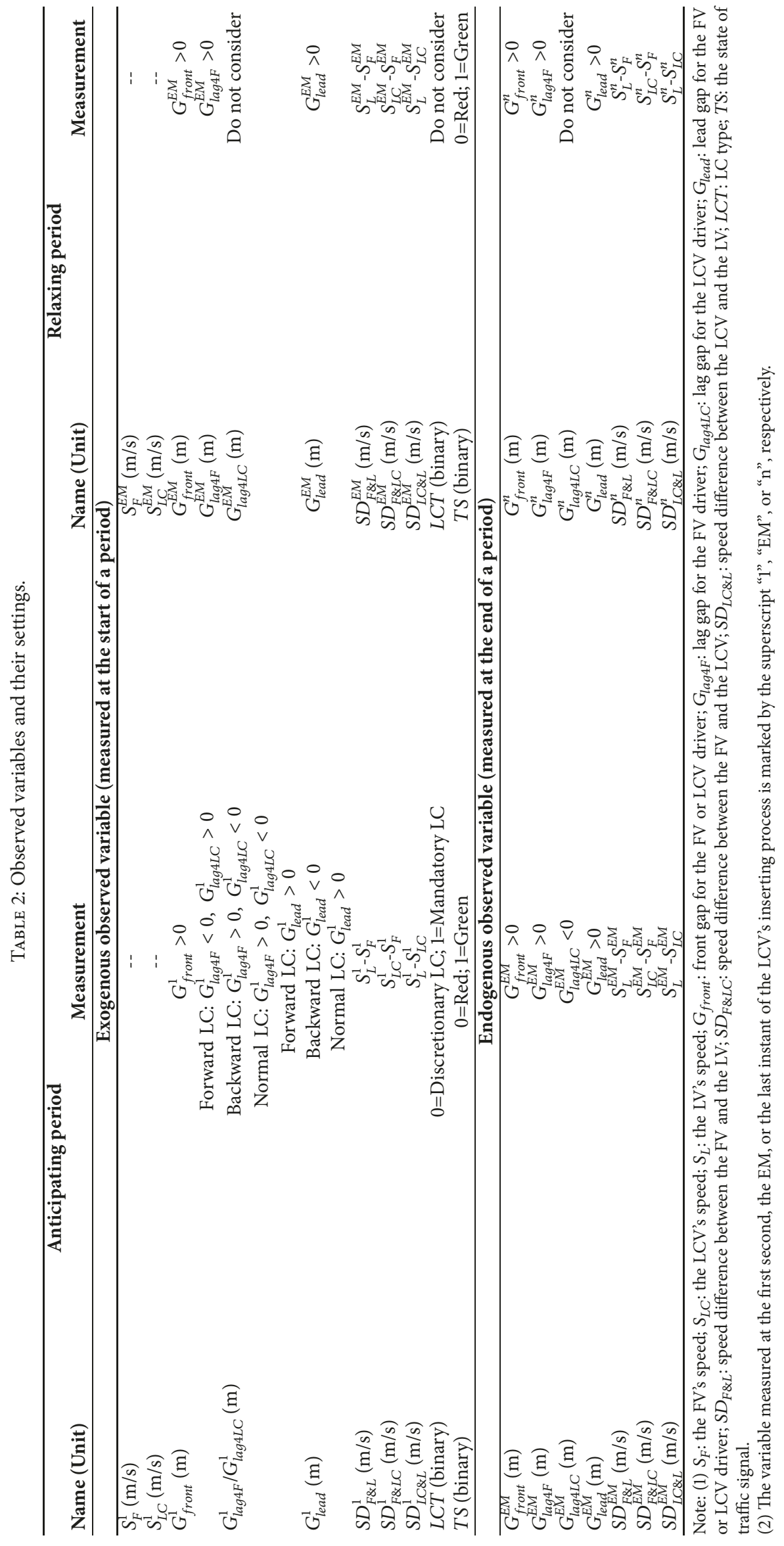


the negative difference happens. At the end of the anticipating period or in the relaxing period, the LCV enters target lane, so each vehicle's position is fixed and the meanings of the gaps do not change for each driver $\left(G_{\text {lag } 4 F}^{E M} / G_{\text {lag } 4 F}^{n}>0, G_{\text {lead }}^{E M} / G_{\text {lead }}^{n}>0\right.$ and $G_{\text {lag } 4 L C}^{E M}<0$ in the table).

3.3. Model Definition. Two SEMs are formulated to specify the conceptual model developed in Section 3.1, and each of them consists of one structural model and two measurement models. Their definitions will be introduced in this section.

3.3.1. Definition of Structural Model. The structural model is to infer whether and how much an external stimuli could affect a driver's anticipations of other vehicles' future movements. The path of transmitting the effect is illustrated in Figure 4 as a directional line pointing from an exogenous observed variable to an endogenous latent variable. The former one models a stimulus while the latter one models the anticipation. Both of the LCV driver and the FV driver are involved in the driving relationships defined in Section 2 and observe the external stimuli in different ways. The two facts are the basis of structural model development.

The FV longitudinally follows the LV on LC target lane, and it also maintains LC relationship in the anticipating period or lateral CF relationship in the relaxing period with the LCV. The anticipations of the FV driver to the developing trends of the two relationships are abstracted as the $L S_{L 4 F}$ and $L S_{L C 4 F}$. The driver also predicts the relative moving trend of the LCV and the LV $\left(L S_{L \& L C 4 F}\right)$. The influences of these three vehicle stimuli on the FV driver are modelled by " $L S_{L 4 F} \longrightarrow L A_{F 2 L}$ " and " $L S_{L C 4 F} / L S_{L \& L C 4 F} \longrightarrow L A_{F 2 L C}$ " in Figures 4(a) and 4(b). In a similar way, the LCV driver's anticipations of future moving trends of the FV and the LV can be modelled as " $L S_{F 4 L C} / L S_{L \& F 4 L C} \longrightarrow L A_{L C 2 F}$ " and " $L S_{L 4 L C} \longrightarrow L A_{L C 2 L}$ " in Figure $4(\mathrm{a})$ and $L S_{L 4 L C} \longrightarrow L A_{L C 2 L}$ in Figure 4(b). Note that the LCV driver is assumed to be immune to the backward influences of the FV any longer in the relaxing period $\left(L S_{F 4 L C}\right.$ and $\left.L S_{L \& F 4 L C}\right)$, as the driver has encroached into the target lane after the EM and occupied a leading position with respect to the FV. Hence, $L S_{F 4 L C}$ and $L S_{L \& F 4 L C}$ are excluded from Figure $4(\mathrm{~b})$. Besides adjacent vehicles' movements, a drivers' anticipation of collision risks is also related to subject vehicle speeds. A higher vehicle speed makes the driver more sensitive to an external stimulus. So speed of the LCV or the PF is assumed to affect its driver's anticipations to other vehicles' movements. These are modelled as " $S_{F}^{1} \longrightarrow L A_{F 2 L} / L A_{F 2 L C}$ " and " $S_{L C}^{1} \longrightarrow$ $L A_{L C 2 F} / L A_{L C 2 L}$ " in Figure $4(\mathrm{a}), " S_{F}^{E M} \longrightarrow L A_{F 2 L} / L A_{F 2 L C}$ " and " $S_{L C}^{E M} \longrightarrow L A_{L C 2 L}$ " in Figure $4(\mathrm{~b})$.

Moreover, LC type (LCT) can reflect the influence of LC intention degree on a driver's behaviour. With respect to a discretionary LC, a mandatory LC usually arouses the LCV driver's stronger LC intention and inserting manoeuvers of the LCV could be more aggressive. Its impact on the FV could be harder too. Such influence is modelled as "LCT $\longrightarrow L A_{F 2 L C} / L A_{L C 2 F}$ " in Figure 4(a). LCT is excluded from Figure 4(b), as the LCV has entered the target lane after the EM and the variance of LC type should be ineffective for the drivers. In addition, the influence of traffic signals (TS) on a driver's decision at urban arterial cannot be ignored. This factor is assumed to affect the drivers' anticipations at the anticipating and relaxing periods. It is modelled as "TS $\longrightarrow$ $L A_{F 2 L} / L A_{F 2 L C} / L A_{L C 2 F} / L A_{L C 2 L}$ " in Figure $4(\mathrm{a})$ and "TS $\longrightarrow$ $L A_{F 2 L} / L A_{F 2 L C} / L A_{L C 2 L}$ " in Figure $4(\mathrm{~b})$.

3.3.2. Definition of Measurement Model. There exist two measurement models in Figures 4(a) and 4(b). One measurement model is used to infer the stimulus of surrounding vehicle perceived by a driver at the start of the anticipating or relaxing period. The stimulus is assumed to derive from vehicle spacing and speed difference, which is modelled as " $L S_{L 4 F} / L S_{L \& F 4 L C} \longrightarrow G_{\text {front }}^{1} / S D_{F \& L}^{1}$ ", " $L S_{L C 4 F} \longrightarrow$ $G_{\text {lag } 4 F}^{1} / S D_{F \& L C}^{1}$ ", " $L S_{L \& L C 4 F} / L S_{L 4 L C} \longrightarrow G_{\text {lead }}^{1} / S D_{L C \& L}^{1}$ ", and " $L S_{F 4 L C} \longrightarrow G_{\text {lag } 4 L C}^{1} S D_{F \& L C}^{1}$ " in Figure $4(\mathrm{a})$ and " $L S_{L 4 F} \longrightarrow G_{\text {front }}^{E M} / S D_{F \& L}^{E M}$ ", " $L S_{L C 4 F} \longrightarrow G_{\text {lag } 4 F}^{E M} / S D_{F \& L C}^{E M}$ ", and " $L S_{L \& L C 4 F} / L S_{L A L C} \longrightarrow G_{\text {lead }}^{E M} / S D_{L C \& L}^{E M}$ " in Figure 4(b). Another measurement model is to identify how the two drivers adjust vehicle spacing and speed difference at the end of a period based on their anticipations of other vehicles' future movements. The FV driver can control its spacing and speed difference to the LV, while the LCV driver can control the ones to the LV. These are modelled as " $L A_{F 2 L} \longrightarrow G_{\text {front }}^{E M} / S D_{F \& L}^{E M}$ " and " $L A_{L C 2 L} \longrightarrow G_{\text {lead }}^{E M} / S D_{L C \& L}^{E M}$ " in Figure $4(\mathrm{a})$ and " $L A_{F 2 L} \longrightarrow G_{\text {front }}^{n} / S D_{F \& L}^{n}$ " and " $L A_{L C 2 L} \longrightarrow G_{\text {lead }}^{n} / S D_{L C \& L}^{n}$ " in Figure 4(b). The drivers of the LCV and the FV jointly control lag gap and the speed difference between them in the anticipating period, so it can be modelled as " $L A_{F 2 L C} \longrightarrow$ $G_{\text {lag } 4 F}^{E M}$ ", " $L A_{L C 2 F} \longrightarrow G_{\text {lag } 4 L C}^{E M}$ ", and " $L A_{F 2 L C} / L A_{L C 2 F} \longrightarrow$ $S D_{F \& L C}^{E M}$ " in Figure 4(a). In the relaxing period, only the FV driver hold the controls, while the LCV driver is assumed to not care the FV's movements any more after the EM. It can be reflected as " $L A_{F 2 L C} \longrightarrow G_{\text {lag } 4 F}^{n} / S D_{F \& L C}^{n}$ " in Figure 4(b).

3.3.3. Correlated Variables. Correlation test is made to the observed variables, and test results are listed in Tables 3 and 4. The bolded coefficients indicate the pairs of the variable whose correlations are modelled in the SEMs, which are illustrated in Figure 4 as the normal rectangles connected by curved lines. The correlations are set based on the logic of driving behaviour. Since the anticipations of the LCV driver and the FV driver are assumed to work simultaneously after the start of anticipating period, the exogenous variables in Figure 4(a) are set to be uncorrelated. At the end of the anticipating and relaxing period, it assumes that the kinematical indicators whose values are determined by the same driver are set to be correlated.

\section{Data Collection}

An arterial link in Shanghai with $200 \mathrm{~m}$ length is selected as the study site. Traffic video was captured at a roadside building in the height of $70 \mathrm{~m}$ to cover the whole link (see Figure 5). The trajectories of 250 group vehicles were extracted from the video by self-developed software [30] in $10 \mathrm{hz}$ and 


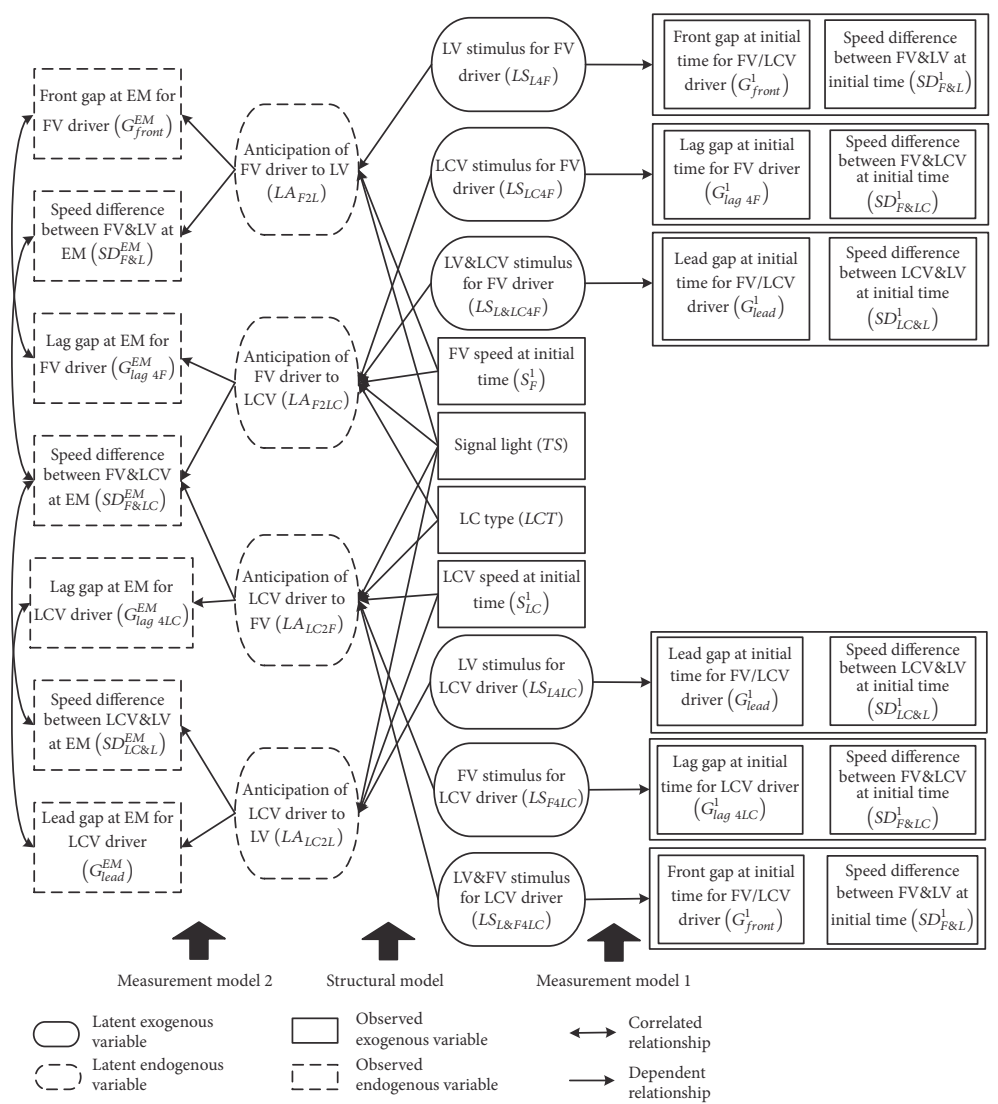

(a) The SEM in the anticipating period

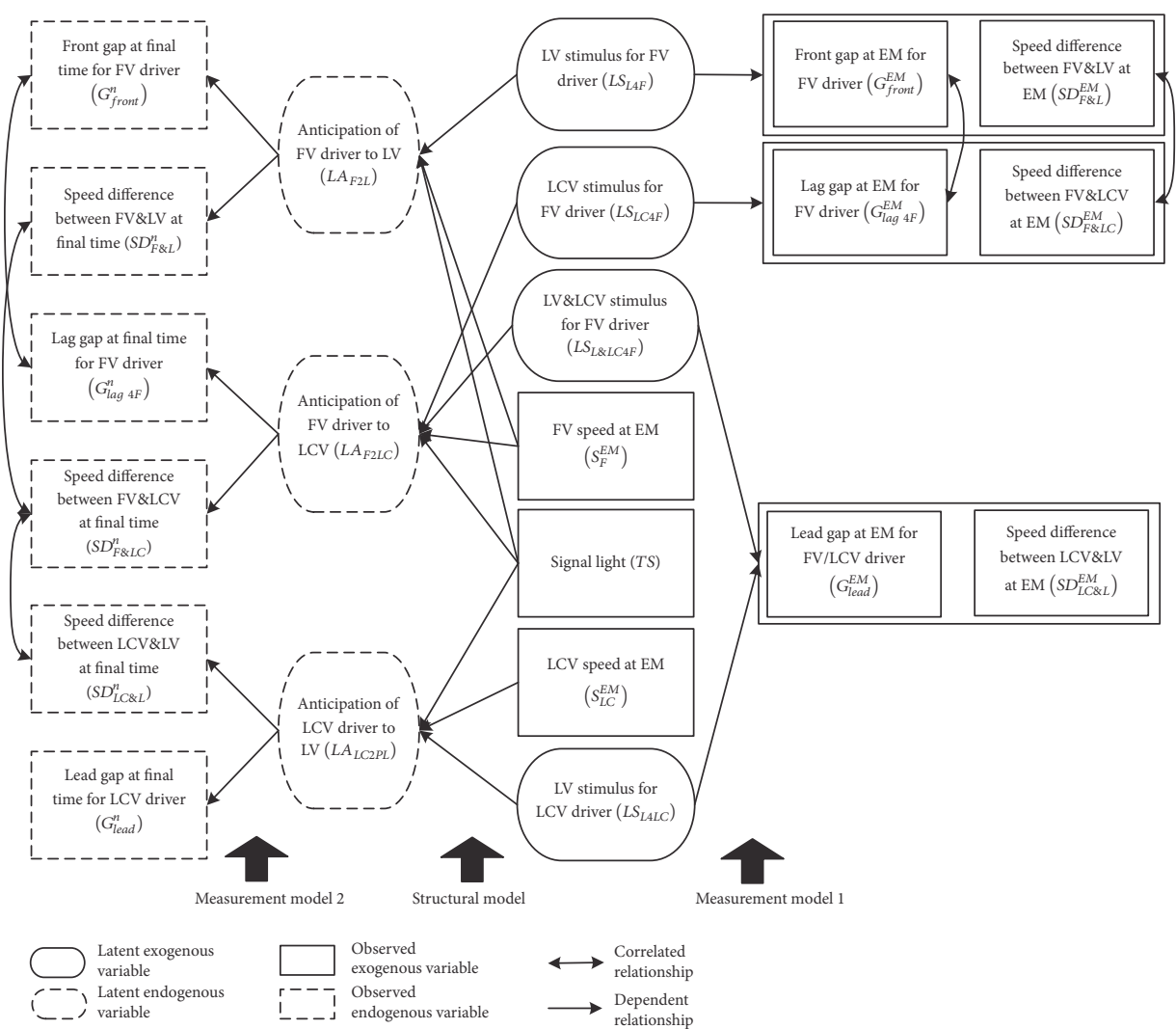

(b) The SEM in the relaxing period

FIGURE 4 


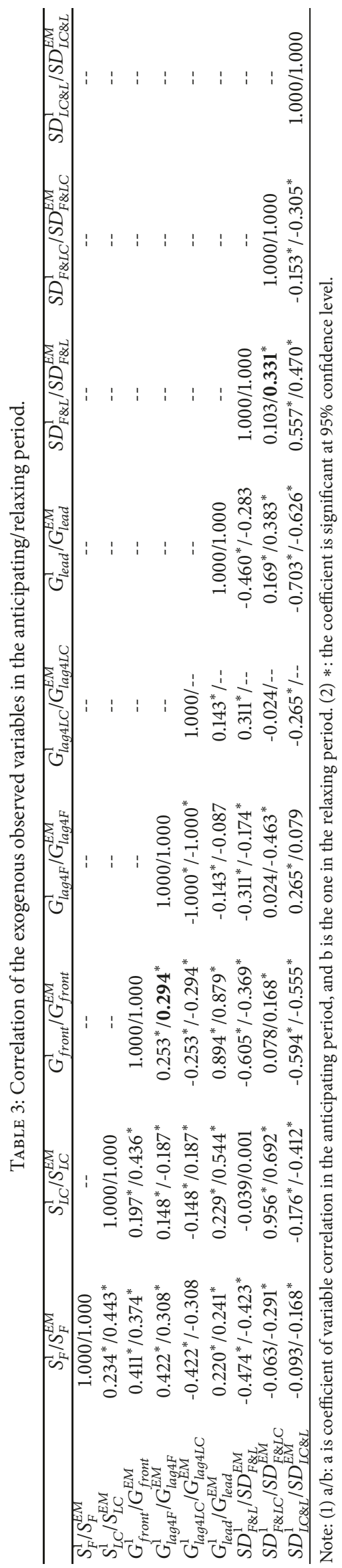




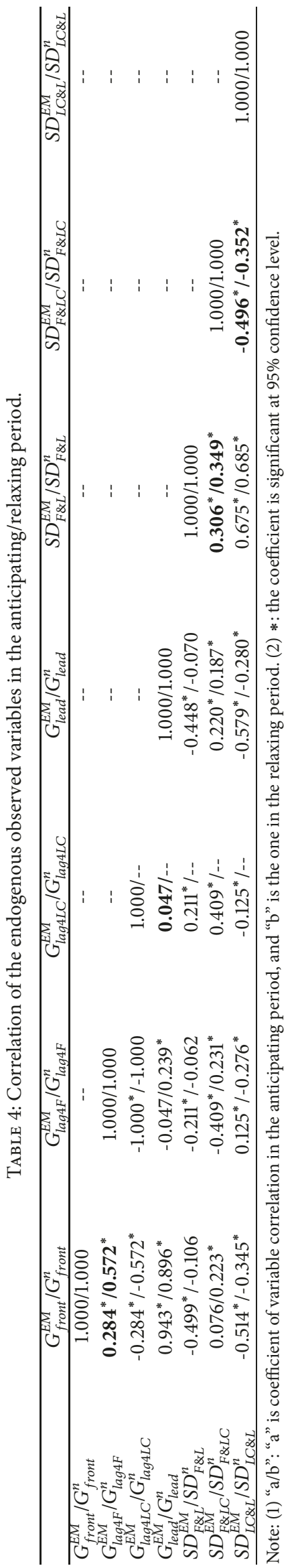




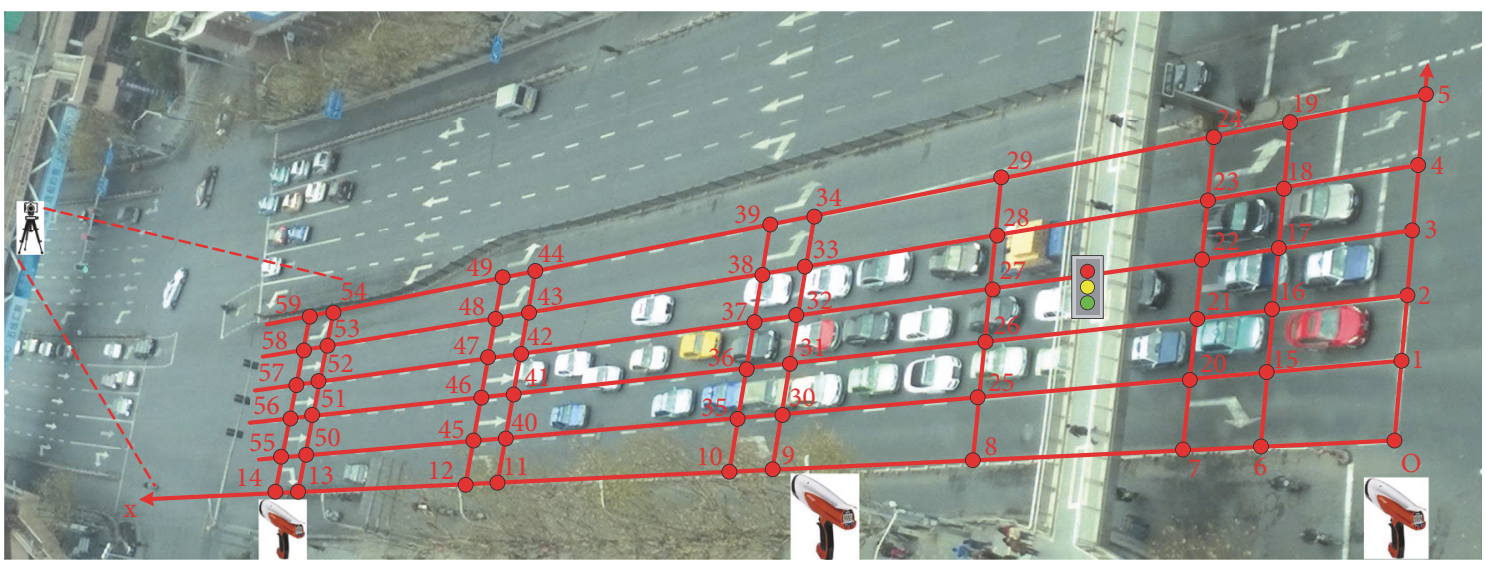

Figure 5: Study site.

calibrated to real-world coordinate using the method in [31]. The trajectory recording points locate at the head or rear bumper of a vehicle, where the subject vehicle collides with other vehicles most possibly. The noise in the raw trajectories was filtered referring to the method in [32]. The speed of some vehicles was calculated from the calibrated trajectories and compared with the values measured by three radar guns located at the cross-sections marked in the figure. The error of the speed calculated from the trajectories was found to be limited in $0.1 \mathrm{~m} / \mathrm{s}$. The vehicle kinematical indicators in Table 2 were calculated from the trajectories and used to estimate the SEMs proposed before. The information of traffic signal and LC type was recorded from a pedestrian overpass at upstream intersection. The LCV's inserting manoeuvre is a discretionary LC if LC original and target lanes serve the same direction traffic; otherwise, it is a mandatory LC.

\section{Results Interpretation and Discussion}

The statistical software Stata is applied to formulate and estimated the two SEMs. The models are estimated at a $95 \%$ confidence level, and the goodness-of-fit measures show they fit the data well: Cronbach alpha value $=0.81 / 0.83$; rootmean-square-error of approximation (RMSEA) $=0.08 / 0.04$; comparative fit index $(\mathrm{CFI})=0.90 / 0.93$; standardized root mean squared residual $(\mathrm{SRMR})=0.07 / 0.05$. Tables 5 and 6 list the estimated unstandardized and standardized coefficients. The former ones will be interpreted in Section 5.1, while the latter ones are used to calculate the indirect path effects listed in Table 7, which will be interpreted in Section 5.2.

\subsection{Model Coefficient}

5.1.1. Structural Model. The estimated coefficients of structural model measure how much the external stimuli appeared at the start of the anticipating and relaxing periods impacts anticipations of the drivers in the FV and the LCV. Results show that an increase of vehicle speed could enhance the drivers' anticipations in both period (" $S_{F}^{1} \longrightarrow$ $L A_{F 2 L} / L A_{F 2 L C}$ " $=0.189 / 0.164>0$ for the FV driver, " $S_{L C}^{1} \longrightarrow$ $L A_{L C 2 F} / L A_{L C 2 L} "=0.146 / 0.149>0$ for the $L C V$ driver in
Table 5; " $S_{F}^{E M} \longrightarrow L A_{F 2 L} / L A_{F 2 L C}$ " $=0.386 / 0.192>0$ for the FV driver, and " $S_{L C}^{E M} \longrightarrow L A_{L C 2 L}$ " $=0.348>0$ for the $L C V$ driver in Table 6). Such a result verifies the authors' speculation that a driver's anticipation of other vehicles' moving trends is related to the subject vehicle's speed. A faster vehicle could increase its driver's sensitivity to external stimuli and impel him or the driver to predict driving condition in the further future. But compared with the speed variance of subject vehicle, other vehicles' stimuli impact the drivers' anticipations heavier (" $L S_{L 4 F} \longrightarrow L A_{F 2 L}$ " $=0.967>0.189=$ " $S_{F}^{1} \longrightarrow L A_{F 2 L}$ " and " $L S_{L C 4 F} \longrightarrow L A_{F 2 L C}$ " $=0.897>0.164=$ " $S_{F}^{1} \longrightarrow L A_{F 2 L C}$ " for the FV driver, " $L S_{F 4 L C} \longrightarrow L A_{L C 2 F}$ " $=0.623>0.146=" S_{L C}^{1} \longrightarrow$ $L A_{L C 2 F}$ " and " $L S_{L 4 L C} \longrightarrow L A_{L C 2 L}$ " $=|0.985|>0.149=$ " $S_{L C}^{1} \longrightarrow L A_{L C 2 L}$ " for the LCV driver in Table 5; " $L S_{L 4 F} \longrightarrow$ $L A_{F 2 L}$ " $=0.753>0.386=$ " $S_{F}^{E M} \longrightarrow L A_{F 2 L}$ " and " $L S_{L C 4 F} \longrightarrow$ $L A_{F 2 L C}$ " $=0.913>0.192=$ " $S_{F}^{E M} \longrightarrow L A_{F 2 L C}$ " for the FV driver, " $L S_{L 4 L C} \longrightarrow L A_{L C 2 L}$ " $=0.858>0.348=$ " $S_{L C}^{E M} \longrightarrow L A_{L C 2 L}$ " for the LCV driver in Table 6). The stimuli come from each driver's perception of vehicle gap or speed difference that exists between (1) subject vehicle and another vehicle and (2) two other vehicles. The stimulus coming from the former vehicle couple is found to impact the drivers' anticipations more than the one of the latter couple at each period (" $L S_{L \& L C 4 F} \longrightarrow L A_{F 2 L C}$ " $=0.232<0.897=" L S_{L C 4 F} \longrightarrow L A_{F 2 L C}$ " for the FV driver, " $L S_{L \& F 4 L C} \longrightarrow L A_{L C 2 F}$ " $=|-0.320|<$ $0.623=$ " $L S_{F 4 L C} \longrightarrow L A_{L C 2 F}$ " for the LCV driver in Table 5; " $L S_{L \& L C 4 F} \longrightarrow L A_{F 2 L C}$ " =| $-0.330 \mid<0.913=$ " $L S_{L C 4 F} \longrightarrow$ $L A_{F 2 L C}$ " for the FV driver in Table 6).

Besides the stimuli of vehicle kinematics, the change of LC type is also found to affect the drivers' anticipations. The LCV driver produces a stronger anticipation in a mandatory LC scenario than in a discretionary one (" $L C T \longrightarrow$ $L A_{L C 2 F}$ " $=0.136$ in Table 5), as the driving task is riskier in the mandatory LC scenario. What is interesting is that the FV driver has a weaker anticipation in a mandatory LC scenario than in a discretionary one ("LCT $\longrightarrow L A_{F 2 L C}$ "=$0.164<0.136=$ " $L C T \longrightarrow L A_{L C 2 F}$ " in Table 5$)$. It could be attributed to the fact that the FV driver can identify the LCV driver's intention more easily in the mandatory LC scenario, but it becomes harder for the driver involved in discretionary 


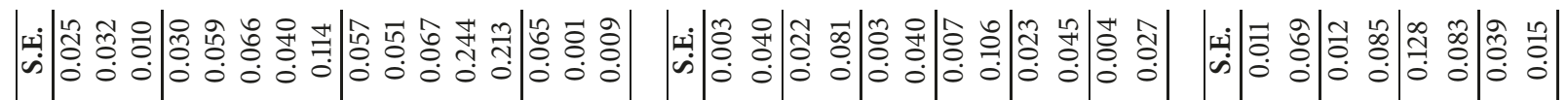

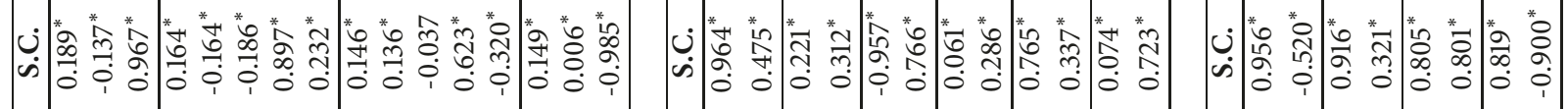

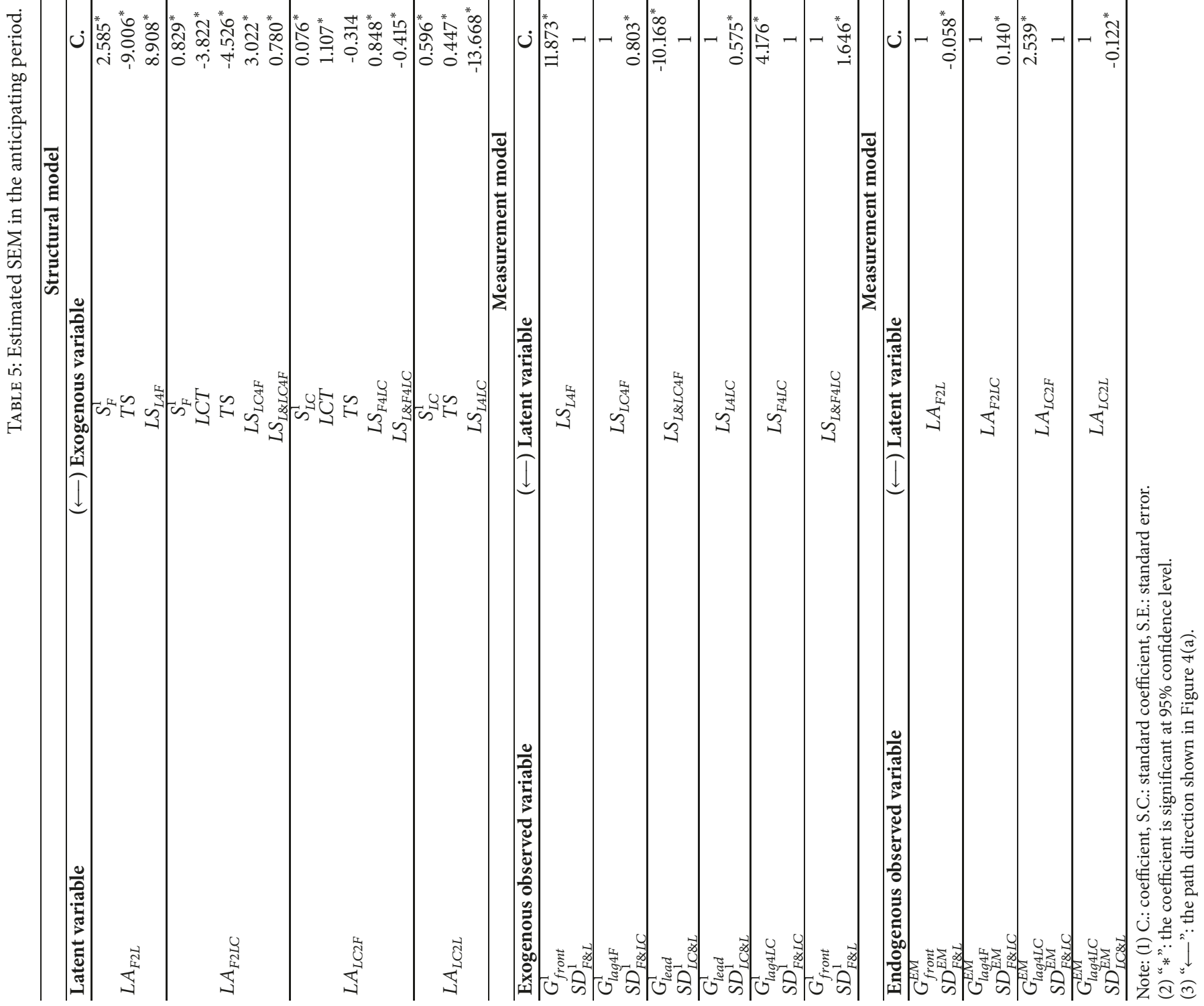




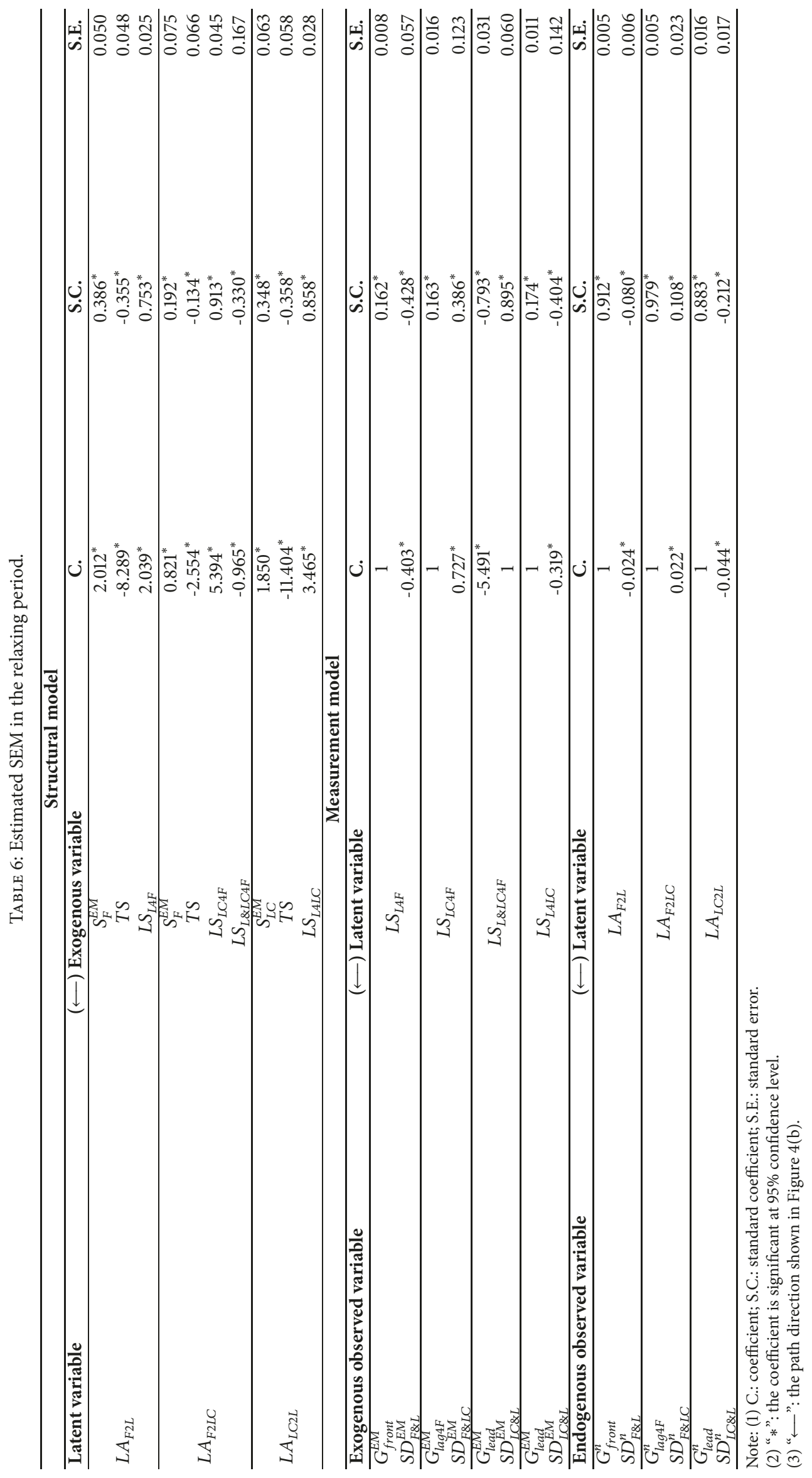




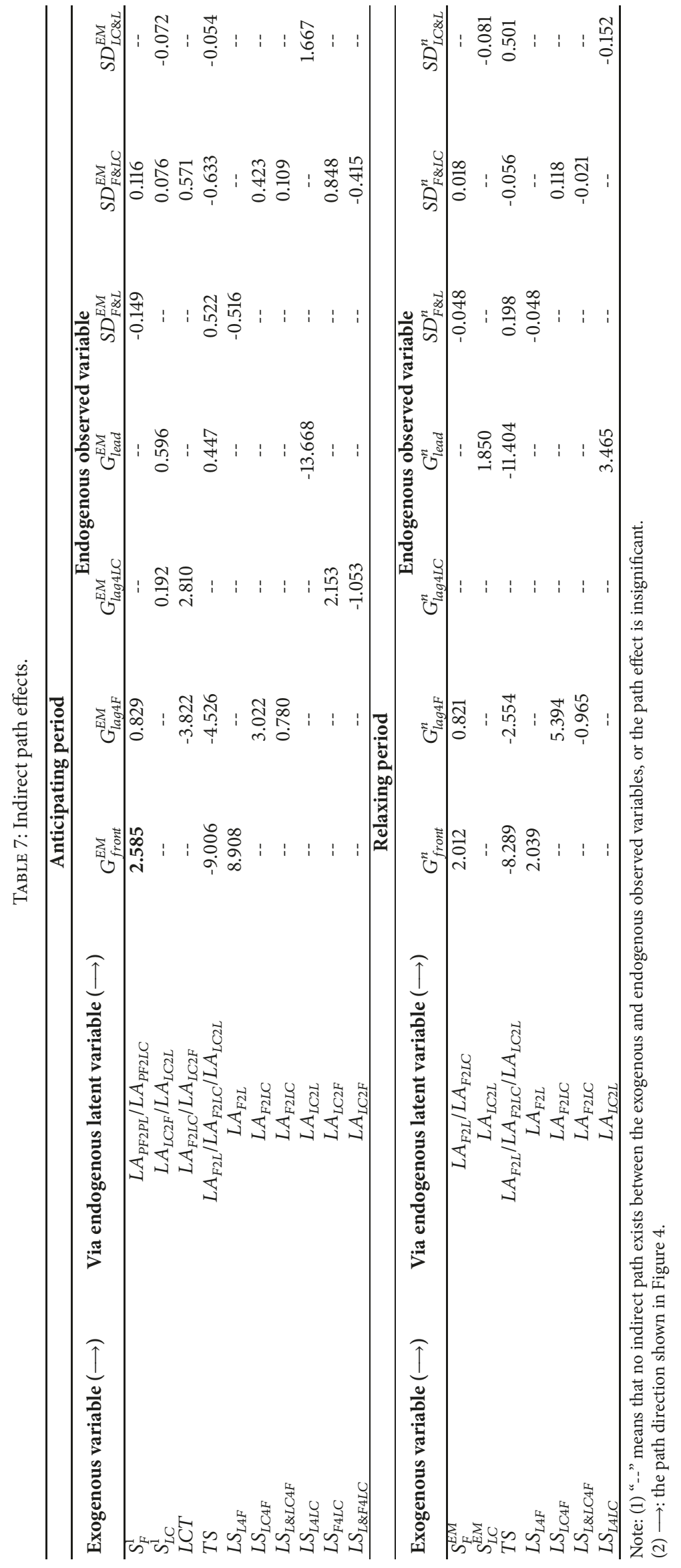


LC scenario. After all, in the latter case the LCV driver can head to the destination without conducting a LC.

We find that the FV driver's anticipation decreases when traffic signal switches from red to green in the two periods ("TS $\longrightarrow L A_{F 2 L} / L A_{F 2 L C}$ " $=-0.137 /-0.186<0$ in Table 5; "TS $\longrightarrow$ $L A_{F 2 L} / L A_{F 2 L C} "=-0.355 /-0.134<0$ in Table 6). A red signal can help the FV driver build a reliable expectation that all vehicles ahead have to decelerate in the near future, so it is easier for the driver to anticipate other vehicles' movements. Obviously, this trend becomes harder to predict in the green phase. In contrast, the LCV driver's anticipation of the FV's movements is hardly affected by the switch of the traffic signal in the anticipating period ("TS $\longrightarrow L A_{L C 2 F}$ " is insignificant in Table 5). Its influence on the LCV driver's anticipation of the LV is much slighter than that of other factors ("TS $\longrightarrow$ $L A_{L C 2 L} "=0.006<" S_{L C}^{1} \longrightarrow L A_{L C 2 L} "=0.149<" L S_{L 4 L C} \longrightarrow$ $L A_{L C 2 L} "=|-0.985|$ in Table 5). The insensitiveness of the LCV driver to the traffic signal in the anticipating period is reasonable, because no matter in the red or green phase, the driver needs to keep safe gap and speed difference with the FV before subject vehicle inserts into target lane. So the LCV driver focuses on predicting the FV's future movements, which is hardly affected by the switch of the traffic signal. When the LCV enters target lane in the relaxing period, traffic signal turns to be influential for the driver ("TS $\longrightarrow$ $L A_{L C 2 L}$ " $=0.358$ in Table 6), as the driver's attention switches from conducting the insertion to following the LV. The future moving trend of the LV becomes a critical issue that needs the LCV driver cares.

5.1.2. Measurement Model. The estimations of two measurement models of the SEMs can tell us (1) which kinematical indicator is major source of vehicle stimuli for a driver at start of a period; (2) which kinematical indicator is mainly adjusted by a driver at end of a period based on his or her anticipation of counterpart's future movements. The results will be interpreted in a temporal sequence from start of the anticipating period to end of the relaxing period.

At the start of the anticipating period, a larger aspect of the LV's stimulus perceived by the FV driver comes from front gap, rather than the speed difference with the LV (" $L S_{L 4 F} \longrightarrow G_{\text {front }}^{1}$ " $=0.964>0.475=" L S_{L 4 F} \longrightarrow S D_{F \& L}^{1}$ " and " $L S_{L \& L C 4 F} \longrightarrow G_{\text {lead }}^{1} "=|-0.957|>0.766=" L S_{L \& L C 4 F} \longrightarrow$ $S D_{L C \& L}^{1}$ " in Table 5). It impels the FV driver to shorten the spacing to the LV. But this adjustment could be weakened by the FV driver's anticipation to the relationship between the LV and the LCV (" $L S_{L \& L C 4 F} \longrightarrow G_{\text {lead }}^{1}$ " $=-0.957<0$ and " $L S_{L 4 F} \longrightarrow G_{\text {front }}^{1} "=0.964>0$ in the table). On the contrary, a larger aspect of the LV's stimulus perceived by the LCV driver derives from vehicle speed difference (" $L S_{L 4 L C} \longrightarrow S D_{L C \& L}^{1}$ " $=0.286>0.061=" L S_{L 4 L C} \longrightarrow G_{\text {lead }}^{1}$ " and " $L S_{L \& F 4 L C} \longrightarrow S D_{F \& L}^{1}$ " $=0.732>0.074=" L S_{L \& F 4 L C} \longrightarrow$ $G_{\text {front }}^{1}$ " in the table). As the LC couple, the FV driver is concerned more about the speed difference to the LCV (" $L S_{L C 4 F} \longrightarrow S D_{F \& L C}^{1}$ " $=0.312>0.221=" L S_{L C 4 F} \longrightarrow G_{\text {lag } 4 F}^{1}$ " in the table), while the LCV driver pays more attention to his or her lag gap with the FV (" $L S_{F 4 L C} \longrightarrow$ $G_{\text {lag } 4 L C}^{1} "=0.765>0.337=" L S_{F 4 L C} \longrightarrow S D_{F \& L C}^{1}$ " in the table).
Then at the end of the anticipating period, based on their anticipations to other vehicles, the drivers of the FV and the LCV adjust their vehicle manoeuvres to reduce collision risk with other vehicles. In addition, their main adjusted kinematical indicators related to the LV are the ones they were concerned of at start of the period. The FV driver cares more about front gap (" $L A_{F 2 L} \longrightarrow G_{\text {front }}^{E M}$ " $=0.956>$ $|-0.520|=" L A_{F 2 L} \longrightarrow S D_{F \& L}^{E M}$ " in Table 5), while the LCV driver cares more about the speed difference with the LV ("LA $A_{L C 2 L} \longrightarrow S D_{L C \& L}^{E M}$ " $=|-0.900|>0.819=" L A_{L C 2 L} \longrightarrow$ $G_{\text {lag } 4 L C}^{E M}$ " in the table). As for their mutual driving relationship, the LCV driver still focuses on adjusting lag gap with the FV (" $L A_{F 2 L C} \longrightarrow G_{\text {lag } 4 F}^{E M}$ " $=0.916>0.321=" L A_{F 2 L C} \longrightarrow$ $S D_{F \& L C}^{E M}$ " in the table), which is also concerned by the driver at the start of this period. However, the FV driver's interest has been changed to lag gap (" $L A_{L C 2 F} \longrightarrow$ $G_{\text {lag } 4 L C}^{E M}$ " $=0.805>0.801=$ " $L A_{L C 2 F} \longrightarrow S D_{F \& L C}^{E M}$ " in the table), rather than the speed difference with the LCV which is the FV driver's main concern at the start of the anticipating period.

An interesting finding is that, in the anticipating period, the drivers' anticipations of their counterparts' movements are affected by the factors differed with the ones they observed at the start of the period. This result could be caused by asymmetric information received by the drivers. Since the LCV driver has a clear target when conducting the insertion, the driver is naturally concerned of the acceptable inserting gap in target lane from the start of the anticipating period. In contrast, the FV driver does not know the LCV driver's intention at the first view in most cases, so the most compelling stimulus for the FV driver is the observable speed difference between the two vehicles. After continuous observations of each other during the anticipating period, both drivers care more about the spacing between them at end of this period. This is because of the fact that lag gap usually achieves the minimum value at the EM, which is the smallest spacing of the two vehicles. Moreover, we find that the drivers' attitudes to the LV are consistent during the anticipating period. It could result of their complete control of the relationships with the LV. They can balance the collision risk to the LV and the one to each other and make the best tactic of adjusting vehicle manoeuvers.

At the start of the relaxing period, both of the FV driver and the LCV driver confirm the LCV's successful insertion, so they follow a similar behavioural logic in this period. The drivers are concerned about the same type of vehicle kinematical indicators at the start of the period, and they adjust the same type of indicators at the end of the period. At the start of the period, speed difference matters more for the drivers than vehicle gap (" $L S_{L 4 F} \longrightarrow S D_{F \& L}^{E M}$ " $=|-0.428|>0.162=" L S_{L 4 F} \longrightarrow$ $G_{\text {front }}^{E M}$ ", " $L S_{L C 4 F} \longrightarrow S D_{F \& L C}^{E M}=0.386>0.163=" L S_{L C 4 F} \longrightarrow$ $G_{\text {lag } 4 F}^{E M}$ ", and " $L S_{L \& L C 4 F} \longrightarrow S D_{L C \& L}^{E M}$ " $=0.895>|-0.793|=$

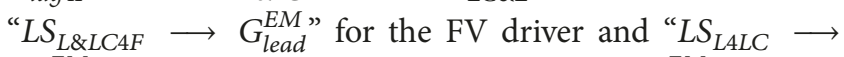
$S D_{L C \& L}^{E M} "=|-0.404|>0.174=" L S_{L 4 L C} \longrightarrow G_{\text {lead }}^{E M}$ " for the LCV driver in Table 6). At end of this period, they adjust vehicle gap more than speed difference (" $L A_{F 2 P L} \longrightarrow$ $G_{\text {front }}^{n} "=0.912>|-0.080|=" L A_{F 2 L} \longrightarrow S D_{F \& L}^{n}$ ", and "LA $A_{F 2 L C} \longrightarrow G_{\text {lag } 4 F}^{n} "=0.979>0.108=" L A_{F 2 L C} \longrightarrow S D_{F \& L C}^{n} "$ 
for the FV driver and " $L A_{L C 2 L} \longrightarrow G_{\text {lead }}^{n}=0.883>|-0.212|=$ " $L A_{L C 2 L} \longrightarrow S D_{L C \& L}^{n}$ " for the LCV driver in the table). In the authors' view, such behavioural similarity of the drivers should be attributed to the following facts. When the drivers confirm the fact that the LCV has entered target lane after the $\mathrm{EM}$, recovering to the desired gap with the leading vehicle becomes their main targets. So vehicle gap is the foremost adjusting indicator. Some studies used this logic to explain the relaxation phenomenon in traffic flow $[24,25]$. To achieve the targets, the vehicle manoeuvre adjustments start from the $\mathrm{EM}$, when the speed difference of the two vehicles is relatively high. So speed difference is the drivers' main stimulus at the $\mathrm{EM}$, and recovery of the desired gaps could be postponed after speed difference decreases.

5.2. Indirect Path Effect. In the SEMs illustrated in Figure 4, some indirect paths connect observed or latent exogenous variables with observed endogenous variables via latent endogenous variables. A path transmits external stimuli each driver perceived at the start of a period via his or her anticipations to the adjusted manoeuvers at the end of the period. The cause-effect paths have physical meanings, so their values are calculated from the unstandardized coefficients. The indirect path effects are interpreted as in Table 7.

Take the effect of the path between initial speed of the $\mathrm{FV}$ and final front gap in the anticipating period (" $S_{F}^{1} \longrightarrow$ $L A_{F 2 L} \longrightarrow G_{\text {front }}^{E M} "=2.585$, bolded in Table 7) as an example. A $1 \mathrm{~m} / \mathrm{s}$ increase of the FV speed at the start of the period could induce its driver leaving an additional $2.585 \mathrm{~m}$ from the $\mathrm{LV}$ at the end of the period. This result is easy to understand. In order to offset the higher collision risk caused by the increased vehicle speed, the FV driver needs to enlarge the front gap to maintain enough emergency braking distance to the LV. The physical meanings of other path effects can be interpreted in this way. To give a big picture about causeeffect relationships between input and output variables of an indirect path, we focus on comparing the estimated effects from a general perspective to obtain knowledge, rather than interpreting the effects one by one.

5.2.1. Different Vehicle Stimuli. We find that when the drivers of the LCV and the FV perceive other vehicles' stimuli, their response could be more severe when adjusting the driving relationships they personally involved $\left(L S_{L C 4 F}\right.$ and $\left.L S_{P F 4 L C}\right)$, than the condition they are only observers $\left(L S_{L \& L C 4 F}\right.$ and $\left.L S_{L \& F 4 L C}\right)$ (" $L S_{L C 4 F} \longrightarrow L A_{F 2 L C} \longrightarrow$ $G_{\text {lag } 4 F}^{E M} / S D_{F \& L C}^{E M}=3.022 / 0.423>0.780 / 0.109=" L S_{L \& L C 4 F} \longrightarrow$ $L A_{F 2 L C} \longrightarrow G_{\text {lag } 4 F}^{E M} / S D_{F \& L C}^{E M}$ " for the FV driver, " $L S_{F 4 L C} \longrightarrow$ $L A_{L C 2 F} \longrightarrow G_{\text {lag } 4 L C}^{E M} / S D_{F \& L C}^{E M}=2.153 / 0.848>|-1.053| / \mid-$ $0.415 \mid=" L S_{L \& F 4 L C} \longrightarrow L A_{L C 2 F} \longrightarrow G_{\text {lag } 4 L C}^{E M} / S D_{F \& L C}^{E M}$ " for the LCV driver in the anticipating period in Table 7; " $L S_{L C 4 F} \longrightarrow$ $L A_{F 2 L C} \longrightarrow G_{\text {lag } 4 F}^{n} / S D_{F \& L C}^{n}=5.394 / 0.118>|-0.965| / \mid-$ $0.021 \mid=" L S_{L \& L C 4 F} \longrightarrow L A_{F 2 L C} \longrightarrow G_{\text {lag } 4 F}^{n} / S D_{F \& L C}^{n}$ " for the $\mathrm{FV}$ driver in the relaxing period in the table). In other words, each driver is more sensitive to the direct vehicle stimuli than to the indirect ones.
For example, when the FV driver perceives a oneunit increase in direct stimulus from the LCV at the start of the anticipating period, the driver's adjustment of lag gap at end of the period is more than the adjustment of lag gap responding to indirect stimulus from the LCV and the LV (" $L S_{L C 4 F} \longrightarrow L A_{F 2 L C} \longrightarrow$ $G_{\text {lag } 4 F}^{E M}$ " $=3.022>0.780=" L S_{L \& L C 4 F} \longrightarrow L A_{F 2 L C} \longrightarrow G_{\text {lag } 4 F}^{E M}$ " in the upper part of Table 7). From the estimation of the measurement model, we know that a one-unit increase of the LCV stimulus for the FV driver derives from a $1 \mathrm{~m}$ increase of initial lag gap (" $L S_{L C 4 F} \longrightarrow G_{\text {lag4F }}^{1}$ "=1 in Table 5), or a 0.803 $\mathrm{m} / \mathrm{s}$ increase of their initial speed difference (" $L S_{L C 4 F} \longrightarrow$ $S D_{F \& L C}^{1} "=0.803$ in Table 5). In contrast, for the indirect stimulus from the interaction between LCV and LV perceived by the FV driver, one unit of its increase is caused by a $10.168 \mathrm{~m}$ decrease of initial lead gap (" $L S_{L \& L C 4 F} \longrightarrow G_{\text {lead }}^{1}$ "=-10.168 in Table 5) or a $1 \mathrm{~m} / \mathrm{s}$ increase of initial speed difference between the LCV and the LV (" $L S_{L \& L C 4 F} \longrightarrow S D_{L C \& L}^{1}$ " $=1$ in Table 5). Since it needs more variants to arouse indirect stimulus $\left(L S_{L \& L C 4 F}\right)$ than direct stimulus $\left(L S_{L C 4 F}\right)(|-10.168|>1$ in gap, $1>0.803$ in speed difference), $L S_{L \& L C 4 F}$ has a relatively smaller effect on the FV driver than $L S_{L C 4 F}$.

In addition, the LCV's direct stimulus for the FV driver could be enhanced in the anticipating period by the indirect stimulus coming from the interaction between the LCV and the $\mathrm{LV}$ (" $L S_{L C 4 F} \longrightarrow L A_{F 2 L C} \longrightarrow G_{\text {lag } 4 F}^{E M} / S D_{F \& L C}^{E M}$ " $=3.022 /$ $0.423>0$, "LS $S_{L \& L C 4 F} \longrightarrow L A_{F 2 L C} \longrightarrow G_{\text {lag } 4 F}^{E M} / S D_{F \& L C}^{E M}$ " $0.780 / 0.109>0$ in the upper part of Table 7). This result indicates that, as a passive acceptor of the LCV's insertion, the FV driver is more sensitive to external stimuli when driving condition is uncertain before the EM. As active executor of the LC movement, the LCV driver is less sensitive than the FV driver before the EM, and direct stimulus of the FV perceived by the LCV driver could be weakened by the indirect stimulus coming from the FV and the $\mathrm{LV}$ (" $L S_{F 4 L C} \longrightarrow L A_{L C 2 F} \longrightarrow G_{\text {lag } 4 L C}^{E M} / S D_{F \& L C}^{E M}$ " $=2.153 / 0.848>0$, " $L S_{F 4 L C} \longrightarrow L A_{L C 2 F} \longrightarrow G_{\text {lag } 4 L C}^{E M} / S D_{F \& L C}^{E M} "=-1.053 /-0.415<0$ in the upper part of the table). Different performances of the two drivers at the anticipating period are determined by asymmetric information received by them. The LCV driver always knows more than the FV driver does about when and where to insert into the target lane. However, such asymmetry disappears after the EM. When the insertion is actually successful, the FV driver performs less sensitively to the external stimuli in the relaxing period than the LCV driver does in the anticipating period. The direct stimulus of the LCV for the FV driver could be weakened by the indirect one from the interaction of the LCV and the LV (" $L S_{L C 4 F} \longrightarrow L A_{F 2 L C} \longrightarrow G_{\text {lag } 4 F}^{n} / S D_{F \& L C}^{n}$ " $=5.394 / 0.118>0$, " $L S_{L \& L C 4 F} \longrightarrow L A_{F 2 L C} \longrightarrow G_{\text {lag } 4 F}^{n} / S D_{F \& L C}^{n} "=-0.965 /-0.021<0$ in the lower part of the table).

There is another interesting finding. In the anticipating period, the responses of the FV driver and the LCV driver to direct stimulus of the counterpart vehicle could be different. The FV driver prefers to keep a larger lag gap when perceiving the LCV stimulus (" $L S_{L C 4 F} \longrightarrow L A_{F 2 L C} \longrightarrow G_{\text {lag } 4 F}^{E M}$ " $=3.022>2.153=" L S_{F 4 L C} \longrightarrow$ $L A_{L C 2 F} \longrightarrow G_{\text {lag } 4 L C}^{E M}$ ” in the upper part of Table 7), while 
the LCV driver is more likely to increase speed difference with the FV (" $L S_{F 4 L C} \longrightarrow L A_{L C 2 F} \longrightarrow S D_{F \& L C}^{E M}=0.848>$ $0.423=" L S_{L C 4 F} \longrightarrow L A_{F 2 L C} \longrightarrow S D_{F \& L C}^{E M}$ " in the upper part of the table). With respect to the LCV driver, the FV driver is more conservative and would always play a risk-averse role in their interactions, e.g., actively decelerating before the EM.

5.2.2. Different Periods. We find that the drivers of the FV and the LCV use different strategies to adjust their vehicle kinematics during the two periods. The FV manoeuver turns to be gentle from the anticipating period to the relaxing period gradually, but the LCV driver's manoeuver performs in an opposite way.

A $1 \mathrm{~m} / \mathrm{s}$ increase of the FV speed $\left(S_{F}^{1} / S_{F}^{E M}\right)$ or the traffic signal switching from red to green $(T S)$, or a one-unit increase of the LV stimulus $\left(L S_{L 4 F}\right)$ could arouse less adjustment of the $\mathrm{FV}$ manoeuvre to the front gap at end of the relaxing period than at the end of the anticipating period (" $S_{F}^{E M} / T S / L S_{L 4 F} \longrightarrow$ $L A_{F 2 L} \longrightarrow G_{\text {front }}^{n}$ " $=2.012 /|-8.289| / 2.039<2.585 /|-9.006| /$ $8.908=$ "S $S_{F}^{1} / T S / L S_{L 4 F} \longrightarrow L A_{F 2 L} \longrightarrow G_{\text {front }}^{E M}$ " in Table 7). Besides, the FV driver also reduces less speed difference with the $\operatorname{LV}\left(S D_{F \& L}^{n}\right)$ or with the $\operatorname{LCV}\left(S D_{F \& L C}^{n}\right)$ in the relaxing period than in the anticipation period (" $S_{F}^{E M} / T S / L S_{L 4 F} \longrightarrow L A_{F 2 L} \longrightarrow S D_{F \& L}^{n}$ " =| - 0.048|/0.198/|$0.048|<|-0.149|/ 0.522 /|-0.516 \mid=" S_{F}^{1} / T S / L S_{L 4 F} \longrightarrow$ $L A_{F 2 L} \longrightarrow S D_{F L}^{E M "}$ and " $S_{F}^{E M} / T S / L S_{L 4 F} \longrightarrow L A_{F 2 L} \longrightarrow$ $S D_{F \& L C}^{n} "=0.018 /|-0.056| / 0.118 /-0.021<0.116 /|-0.633| / 0.432 /$ $0.109=$ "S $S_{F}^{1} / T S / L S_{L 4 F} \longrightarrow L A_{F 2 L} \longrightarrow S D_{F \& L C}^{E M}$ " in the table). The only exception happens when the FV driver adjusts the lag gap with the LCV. With increased vehicle speed or change of traffic signal, the FV driver conducts more adjustment of the lag gap in the anticipating period than in the relaxing period " $S_{F}^{1} / T S \longrightarrow L A_{F 2 L C} \longrightarrow$ $G_{\text {lag } 4 F}^{E M} "=0.829 /|-4.526|>0.821 /|-2.554|=" S_{F}^{E M} / T S \longrightarrow$ $L A_{F 2 L C} \longrightarrow G_{\text {lag } 4 F}^{n}$ " in the table). However, with a oneunit increase of LCV-involved stimulus, the FV driver could execute less adjustment of the lag gap in the anticipating period (" $L S_{L C 4 F} / L S_{L \& L C 4 F} \longrightarrow L A_{F 2 L C} \longrightarrow$ $G_{\text {lag } 4 F}^{n} "=5.394 /|-0.965|>3.022 / 0.780=" L S_{L C 4 F} / L S_{L \& L C 4 F} \longrightarrow$ $L A_{F 2 L C} \longrightarrow G_{\text {lag } 4 F}^{E M}$ ”). This exception reflects a feature of the FV driver's behaviour: the driver can tolerate a small gap with the LCV temporally when the LCV tries to insert into target lane in the anticipating period and recovers to the desired following spacing with the LCV after it enters target lane in the relaxing period. This feature is also found by previous studies $[21,26]$. It means that, in the relaxing period, the FV could recover desired lag gap to the LCV in a gentler way than it does in the anticipating period by maintaining less speed differences with the LV and the LCV.

In addition, the LCV driver is found to have a stronger intention to narrow speed difference with the $\mathrm{LV}$ in the relaxing period than in the anticipating period $\left(" S_{L C}^{E M} / L S_{L 4 L C} \longrightarrow L A_{L C 2 L} \longrightarrow S D_{L C \& L}^{n}\right.$ "=-0.081/-0.152< $-0.072 / 1.667=$ " $S_{L C}^{1} / L S_{L A L C} \longrightarrow L A_{L C 2 L} \longrightarrow S D_{L C \& L}^{E M}$ " in Table 7). However, the LCV driver has to maintain a larger lead gap in the relaxing period than in the anticipating period $\left(" S_{L C}^{E M} / L S_{L 4 L C} \longrightarrow L A_{L C 2 L} \longrightarrow G_{\text {lead }}^{n}\right.$ " $=1.850 / 3.465>0.596 /$ $-13.668=$ " $S_{L C}^{1} / L S_{L A L C} \longrightarrow L A_{L C 2 L} \longrightarrow G_{\text {lead }}^{E M "}$ in the table). This result could be caused by speed advantage of the LV with respect to the LCV. Since the LV is hardly affected by the vehicles behind it, its speed could be higher than the LCV and the FV in most part of the LCV's inserting process, especially heavy interactions existing between the LCV and the FV in the anticipating period. In the limited time of the relaxing period, the LCV driver is difficult to shorten lead gap with the LV. Even if the gap is shortened, the speed difference between the two vehicles is enlarged ("TS $\longrightarrow L A_{L C 2 L} \longrightarrow$ $G_{\text {lead }}^{n} / S D_{L C \& L}^{n} "=-11.404 / 0.501$ in the table). Recovering to the desired gap with the LV could take the LCV driver a relatively long time.

5.2.3. Traffic Signal. The influence of traffic signal on a driver's behaviour is verified by study results. We find that the manoeuvre adjustments of the FV and the LCV at the end of a period could be affected by the switch of traffic signal. In some cases, this influence is even stronger than that of vehicle stimulus. For example, when traffic signal switches from red to green, the FV leaves a shorter front gap at end of the anticipating period or relaxing period ("TS $\longrightarrow L A_{F 2 L} \longrightarrow$ $G_{\text {front }}^{E M}$ " $=-9.006<0$ and "TS $\longrightarrow L A_{F 2 L} \longrightarrow G_{\text {front }}^{n}$ "=$8.289<0$ in Table 7 ). The shortened spacing outweighs the increased one of the front gap caused by the LV's stimulus ("TS $\longrightarrow L A_{F 2 L} \longrightarrow G_{\text {front }}^{E M} "=|-9.006|>8.908=" L S_{L 4 F} \longrightarrow$ $L A_{F 2 L} \longrightarrow G_{\text {front }}^{E M}$ " and "TS $\longrightarrow L A_{F 2 L} \longrightarrow G_{\text {front }}^{n}$ "=1 8.289| $>2.039=" L S_{L 4 F} \longrightarrow L A_{F 2 L} \longrightarrow G_{\text {front }}^{n}$ " in the table). Similar influences can also be found in the variance of other vehicle kinematical indicator ("TS $\longrightarrow L A_{F 2 L C} \longrightarrow$ $G_{\text {lag } 4 F}^{E M} "=|-4.526|>3.022=" L S_{L C 4 F} \longrightarrow L A_{F 2 L C} \longrightarrow G_{\text {lag } 4 F}^{E M}$ ", “TS $\longrightarrow L A_{L C 2 L} \longrightarrow G_{\text {lead }}^{n}$ " $=|-11.404|>3.465=" L S_{L 4 L C} \longrightarrow$ $L A_{L C 2 L} \longrightarrow G_{\text {lead }}^{n}$ ", “TS $\longrightarrow L A_{F 2 L} \longrightarrow S D_{F \& L}^{n} "=0.198>$ $|-0.048|=" L S_{L A F} \longrightarrow L A_{F 2 L} \longrightarrow S D_{F \& L}^{n}$ ", and "TS $\longrightarrow$ $L A_{L C 2 L} \longrightarrow S D_{L C \& L}^{n} "=0.501>|-0.152|=" L S_{L 4 L C} \longrightarrow$ $L A_{L C 2 L} \longrightarrow S D_{L C \& L}^{n}$ " in the table). This finding reminds us of the necessity of considering the role of traffic signals in the analysis of drivers' psychology and behaviour, though this issue is rarely addressed in existing studies [33]. In real traffic, the driver receives much dynamic information, such as the instructions provided by traffic control devices, vehicle or roadside lighting, etc. For instance, rear braking light and turning light of the vehicle could deliver a clear message about subject driver's intention to the surrounding drivers, while traffic signals indicate the right of way in specific traffic movement. All of such information could affect the driver's anticipations of other vehicles' movements, so they should be considered in the models simulating the LC or CF behaviour during the LCV's inserting process.

\section{Conclusions}

This study analyses the working mechanism of anticipating ability of the drivers in the LCV's inserting process in virtue of SEM. The developed two SEMs are calibrated with data extracted from vehicle trajectories and manual observations. 
We find that (1) increasing the speed of the FV or the LCV could impel subject driver to anticipate driving condition in the further future, but the stimuli are lower than the ones the driver perceives by comparing the kinematics of subject vehicle with other vehicles; (2) the variance of vehicle gap or speed difference could affect the anticipations of the LCV driver and the FV driver, but which factor is the main source for a driver depends on the driver's role in the anticipating or relaxing period; (3) the drivers care more about the stimulus coming from the vehicle interaction he or she is involved in than the one he or she only observes; (4) the FV driver makes gentler adjustment of vehicle manoeuvre in the relaxing period than in the anticipating period, while the LCV driver acts in the opposite way; (5) the drivers' anticipations of other vehicles' movements could be affected by the change of LC type and traffic signal, and they adjust vehicle manoeuvres in different ways.

The findings of this study can be used to improve the simulation accuracy of LC traffic. For instance, the parameters of LC model or CF model should be adjusted in a timedependent way, as this study finds that the main kinematical indicators each driver cares about could vary with his/her role in a specific LC stage. Another promising application is to consider the drivers' anticipating ability into motion planning algorithm of autonomous vehicle [34-36]. One of the most challenges in this field is to predict a human driver's response to the movements of autonomous vehicle, and this task could demand a lot of sensing and computing resources of the vehicle. If the human anticipating ability in complex driving circumstance, such as the LCV executing insertion in urban traffic, is considered in the algorithm of autonomous vehicle, it could help release the sensing and computing burden.

Nonetheless, this study leaves ample room for future improvements. First, since vehicle kinematical indicators are recorded only at the start or end of a period, the influence of driver's anticipation on his or her behaviours is estimated on a temporal average level. In fact, such ability keeps working during the whole LCV's inserting process. Future studies should take dynamics of the drivers' anticipations into account and produce a more accurate estimation of this influence. In addition, only the cases of successful LC are analysed in this study, so the length of the inserting process is limited. Sometimes the insertion is time-consuming or even unsuccessful $[8,9]$. These cases should be considered in future study. Finally, only three vehicles directly involved in the inserting process are analysed here. However, more surrounding vehicles could affect or be affected by the LCV, such as its follower in the original lane. Their responses to the inserting movement are related to the LCV's impact on traffic flow, so the analysis of their manoeuvres could improve our understanding of traffic flow stability in LC scenario.

\section{Appendix}

\section{Study Period}

The start of the anticipating period shown in Figure 1(b) (instant 1) is defined according to relative positions of the LCV and the FV in the three scenarios shown in Figure 3.
(1) In the forward inserting scenario (see Figure 3(a)), the LCV intends to overtake the FV and find opportunity to conduct an LC. Instant 1 refers to the moment that head bumpers of the LCV and the FV align, because from this moment the FV driver definitely notices the LCV and begins to anticipate its future movements.

(2) In the backward inserting scenario (see Figure 3(b)), instant 1 is defined as the moment when the vehicles' back bumpers align.

(3) In the median inserting scenario (see Figure 3(c)), the LCV has run aside the gap between the LV and the FV for a while, and the variance of its acceleration could be too slight to catch the FV driver's attention. So instant 1 is defined as the moment when the LCV starts its lateral movement, or its turn signal is activated that can be seen by the FV driver.

It is noteworthy that the definitions of instant 1 above are set to ensure the LCV and the FV drivers' anticipation being working at that moment. In most cases, the LCV driver predicts the FV's movements in advance. To analyse the two drivers' interactive anticipations, the start of anticipating period should be defined as the moment the FV driver definitely anticipates the LCV's movement as well. After instant 1 , the LCV driver usually synchronizes his or her vehicle speed along with that of the FV $[16,17]$ until finding an acceptable gap to cut in.

The anticipating period ends at the EM and the relaxing period starts then, even though the LCV's insertion has not ended. Such a definition which derives from a fact that both of the LCV and the FV drivers realize the LCV has entered target lane successfully after the EM. The relaxing period is assumed to end when the LCV's body is back to parallel to lane marker line of the target lane (instant 3 in Figures 1(a) and 1(b)). The relaxing period defined here could be shorter than the one defined in other studies [21], in which the period does not end until the FV recovers to its desired space with the LCV (instant 4 in Figure 1(b)). This paper does not follow the previous definition, as the study scenario here is urban arterial, rather than the freeway in those studies. Due to the disturbance of traffic signals and complex driving circumstances in urban road, driver's desired space with his or her leading vehicle should be redefined. For instance, drivers in a discharging flow at a green phase or the ones in a queuing flow at a red phase could find it difficult to keep a steady desire space. That is why the relaxing period assumes to end when the LCV's inserting movement is finished.

\section{Data Availability}

The funding agency does not allow the data to be open to the public without their permission.

\section{Conflicts of Interest}

The authors declare that they have no conflicts of interest.

\section{Acknowledgments}

This work was supported by the National Key Research and Development Program of China (Grant no. 2018YFB0105104), 
the National Natural Science Foundation of China (Grants nos. 51505037 and 71701031), the Key Research and Development Program of Shaanxi Province (Grants nos. 2018ZDCXLGY-05-04, 2018ZDCXL-GY-05-07-02, and 2018ZDXM-GY013), and the Fundamental Research Funds for the Central Universities (Grants nos. 300102328101, 300102328401, 300102328205, and 300102328501).

\section{References}

[1] J. Weng, G. Du, D. Li, and Y. Yu, “Time-varying mixed logit model for vehicle merging behavior in work zone merging areas," Accident Analysis \& Prevention, vol. 117, pp. 328-339, 2018.

[2] H. Park, C. Oh, J. Moon, and S. Kim, "Development of a lane change risk index using vehicle trajectory data," Accident Analysis \& Prevention, vol. 110, pp. 1-8, 2018.

[3] R. Thijssen, T. Hofman, and J. Ham, "Ecodriving acceptance: An experimental study on anticipation behavior of truck drivers," Transportation Research Part F: Traffic Psychology and Behaviour, vol. 22, pp. 249-260, 2014.

[4] P. Hofmann, G. Rinkenauer, and D. Gude, "Preparing lane changes while driving in a fixed-base simulator: Effects of advance information about direction and amplitude on reaction time and steering kinematics," Transportation Research Part F: Traffic Psychology and Behaviour, vol. 13, no. 4, pp. 255-268, 2010.

[5] E. Chan, A. K. Pradhan, A. Pollatsek, M. A. Knodler, and D. L. Fisher, "Are driving simulators effective tools for evaluating novice drivers' hazard anticipation, speed management, and attention maintenance skills?" Transportation Research Part F: Traffic Psychology and Behaviour, vol. 13, no. 5, pp. 343-353, 2010.

[6] Z. Zheng, "Recent developments and research needs in modeling lane changing," Transportation Research Part B: Methodological, vol. 60, pp. 16-32, 2014.

[7] Y. Xi and M. Crisler, "A review of lane change definitions and identification methods," in Proceedings of the 92nd Transportation Research Board Annual Meeting, Transportation Research Board of the National Academies, Washington, D.C, 2013.

[8] J. Sun, J. Ouyang, and J. Yang, "Modeling and analysis of merging behavior at expressway on-ramp bottlenecks," Transportation Research Record, vol. 2421, pp. 74-81, 2014.

[9] L. Zhao, J. Sun, and H. Zhang, "Observations and analysis of multistep-approach lane changes at expressway merge bottlenecks in shanghai, China," Transportation Research Record, no. 2395, pp. 73-82, 2013.

[10] A. Kesting, M. Treiber, and D. Helbing, "General lane-changing model MOBIL for car-following models," Transportation Research Record: Journal of the Transportation Research Board, vol. 1999, no. 1, pp. 86-94, 2007.

[11] C. F. Choudhury, V. Ramanujam, and M. E. Ben-Akiva, "Modeling acceleration decisions for freeway merges," Transportation Research Record: Journal of the Transportation Research Board, no. 2124, pp. 45-57, 2009.

[12] C. F. Choudhury and M. E. Ben-Akiva, "Modelling driving decisions: A latent plan approach," Transportmetrica A: Transport Science, vol. 9, no. 6, pp. 546-566, 2013.

[13] W. Schakel, V. Knoop, and B. Van Arem, "Integrated lane change model with relaxation and synchronization," Transportation Research Record, vol. 2316, pp. 47-57, 2012.
[14] A. Kesting, M. Treiber, M. Schönhof, and D. Helbing, "Extending adaptive cruise control to adaptive driving strategies," Transportation Research Record, no. 2000, pp. 16-24, 2007.

[15] D. Yang, L. Zhu, B. Ran, Y. Pu, and P. Hui, "Modeling and Analysis of the Lane-Changing Execution in Longitudinal Direction," IEEE Transactions on Intelligent Transportation Systems, vol. 17, no. 10, pp. 2984-2992, 2016.

[16] X. Wan, P. Jin, L. Zheng, Y. Cheng, and B. Ran, "Speed synchronization process of merging vehicles from the entrance ramp," Transportation Research Record, no. 2391, pp. 11-21, 2013.

[17] X. Wan, P. J. Jin, F. Yang, and B. Ran, "Merging preparation behavior of drivers: How they choose and approach their merge positions at a congested weaving area," Journal of Transportation Engineering, vol. 142, no. 9, Article ID 05016005, 2016.

[18] D. Ngoduy, "Macroscopic effects of multianticipative driving behavior on traffic flow characteristics," Transportation Research Record: Journal of the Transportation Research Board, no. 2124, pp. 103-112, 2009.

[19] N. Farhi, H. Haj-Salem, and J.-P. Lebacque, "Multianticipative piecewise-linear car-following model," Transportation Research Record, vol. 2315, no. 1, pp. 100-109, 2012.

[20] B. Gunay, "Car following theory with lateral discomfort," Transportation Research Part B: Methodological, vol. 41, no. 7, pp. 722735, 2007.

[21] Z. Zheng, S. Ahn, D. Chen, and J. Laval, "The effects of lanechanging on the immediate follower: anticipation, relaxation, and change in driver characteristics," Transportation Research Part C: Emerging Technologies, vol. 26, pp. 367-379, 2013.

[22] C. F. Choudhury and M. M. Islam, "Modelling acceleration decisions in traffic streams with weak lane discipline: A latent leader approach," Transportation Research Part C: Emerging Technologies, vol. 67, pp. 214-226, 2016.

[23] A. Duret, S. Ahn, and C. Buisson, "Passing rates to measure relaxation and impact of lane-changing in congestion," Computer-Aided Civil and Infrastructure Engineering, vol. 26, no. 4, pp. 285-297, 2011.

[24] S. Kim and B. Coifman, "Driver relaxation impacts on bottleneck activation, capacity, and the fundamental relationship," Transportation Research Part C: Emerging Technologies, vol. 36, pp. 564-580, 2013.

[25] C. Appert-Rolland and J. Du Boisberranger, "Macroscopic relaxation after on-ramps in real data and in cellular automata simulations," Transportation Research Part C: Emerging Technologies, vol. 34, pp. 162-175, 2013.

[26] P. Hidas, "Modelling vehicle interactions in microscopic simulation of merging and weaving," Transportation Research Part C: Emerging Technologies, vol. 13, no. 1, pp. 37-62, 2005.

[27] R. H. Hoyle, Handbook of structural equation modeling, The Guilford Press, New York, NY, USA, 2012.

[28] T. Ram and K. Chand, "Effect of drivers' risk perception and perception of driving tasks on road safety attitude," Transportation Research Part F: Traffic Psychology and Behaviour, vol. 42, pp. 162-176, 2016.

[29] V. L. Knoop, M. Keyvan-Ekbatani, M. de Baat, H. Taale, and S. P. Hoogendoorn, "Lane Change Behavior on Freeways: An Online Survey Using Video Clips," Journal of Advanced Transportation, vol. 2018, Article ID 9236028, 11 pages, 2018.

[30] L. Zhang, H. Wang, and L. Li, "Vehicle detection and tracking in video from moving airborne platform," Journal of Computational Information Systems, vol. 10, no. 1, pp. 1-8, 2014. 
[31] K. Ismail, T. Sayed, and N. Saunier, "A methodology for precise camera calibration for data collection applications in urban traffic scenes," Canadian Journal of Civil Engineering, vol. 40, no. 1, pp. 57-67, 2013.

[32] M. Montanino and V. Punzo, “Trajectory data reconstruction and simulation-based validation against macroscopic traffic patterns," Transportation Research Part B: Methodological, vol. 80, pp. 82-106, 2015.

[33] J. Barceló, M. Kuwahara, and M. Miska, "Traffic Data Collection and Its Standardization," in Traffic Data Collection and its Standardization, vol. 144 of International Series in Operations Research \& Management Science, pp. 1-10, Springer New York, New York, NY, 2010.

[34] Z. Xu, K. Zhang, H. Min, Z. Wang, X. Zhao, and P. Liu, "What drives people to accept automated vehicles? Findings from a field experiment," Transportation Research Part C: Emerging Technologies, vol. 95, no. 10, pp. 320-334, 2018.

[35] Z. Xu, M. Wang, F. Zhang, S. Jin, J. Zhang, and X. Zhao, "PaTAVTT: A Hardware-in-the-Loop Scaled Platform for Testing Autonomous Vehicle Trajectory Tracking," Journal of Advanced Transportation, vol. 2017, Article ID 9203251, 2017.

[36] Y. Liu, J. Guo, J. Taplin, and Y. Wang, "Characteristic analysis of mixed traffic flow of regular and autonomous vehicles using cellular automata," Journal of Advanced Transportation, vol. 2017, Article ID 8142074, 2017. 


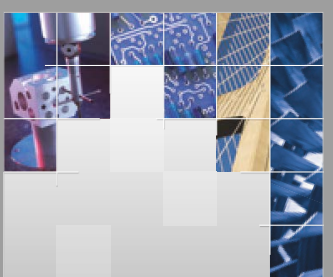

\section{Enfincering}
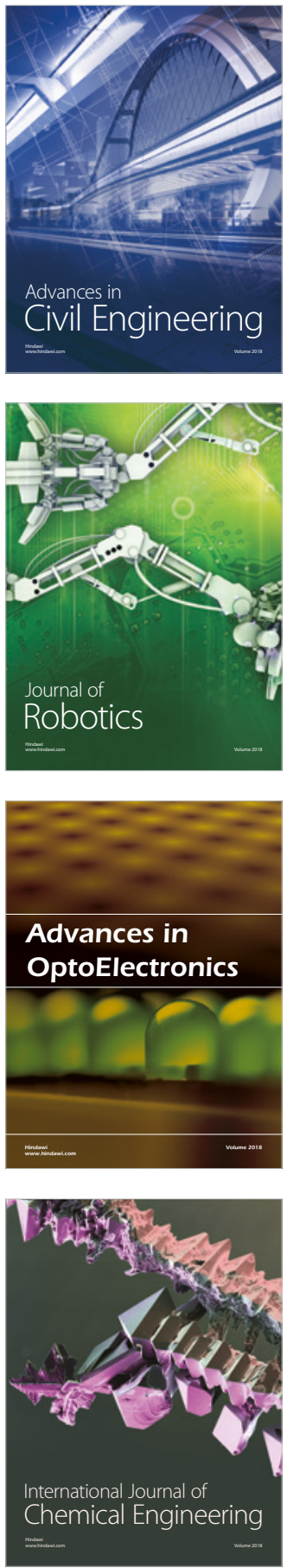

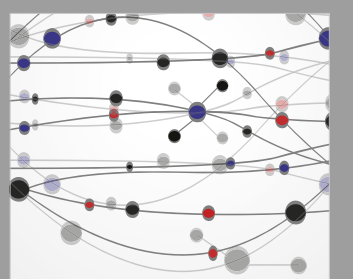

\section{Rotating \\ Machinery}

The Scientific World Journal

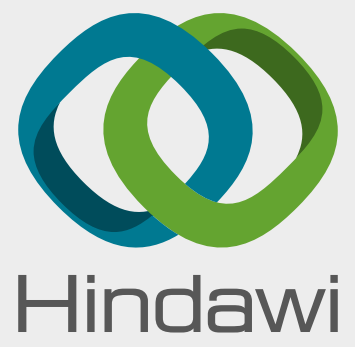

Submit your manuscripts at

www.hindawi.com
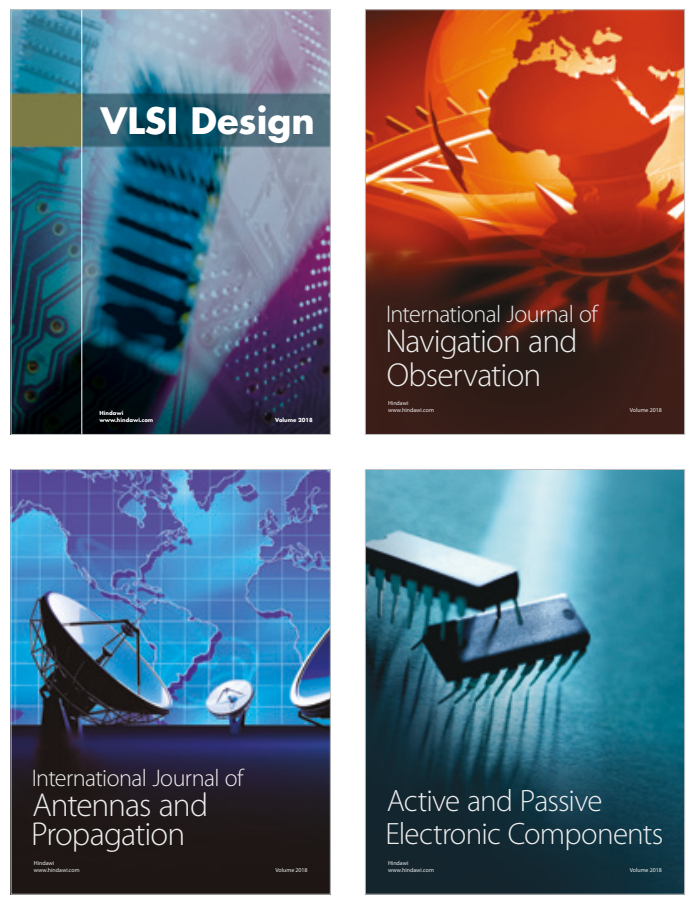
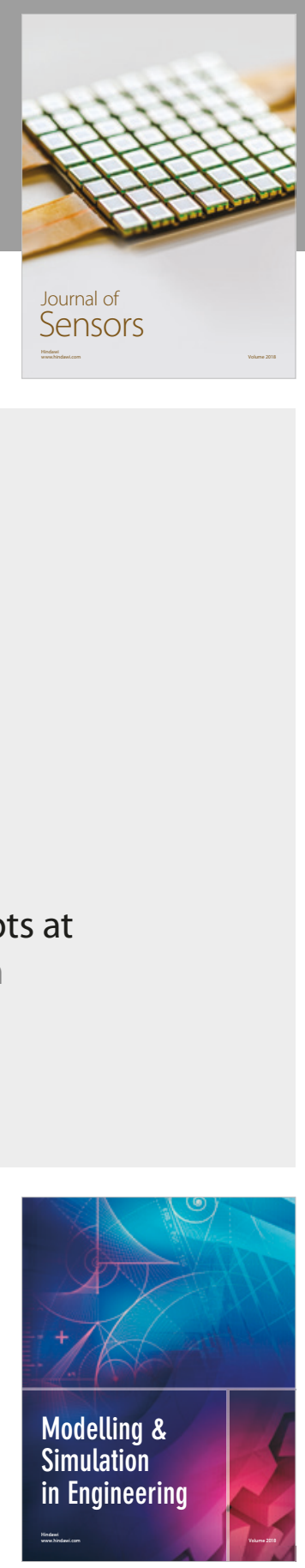

\section{Advances \\ Multimedia}
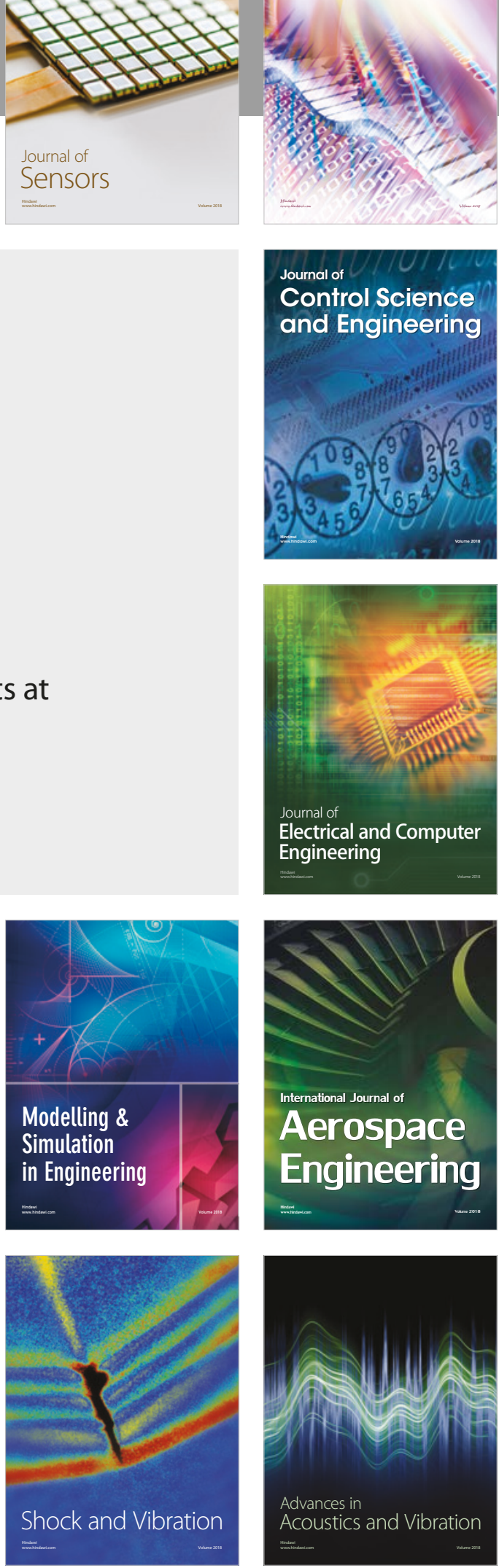